\title{
Investigation of Flame Stretch in Turbulent Lifted Jet Flame
}

\author{
S.Ruan $^{1}$, N.Swaminathan ${ }^{1, *}$, Y.Mizobuchi ${ }^{2}$
}

1 Engineering Department, Cambridge University, Trumpington Street, Cambridge CB2 1PZ, UK.

2 Aerospace Research and Development Directorate, Japan Aerospace Exploration Agency, Jindaiji-Higashimachi Chofu, Tokyo 182-8522, Japan.

${ }^{*}$ Corresponding author: N. Swaminthan. E-mail: ns341@ cam.ac.uk

Submitted for publication in Combustion Science and Technology

(October 17, 2013) 


\section{Abstract}

DNS data of a laboratory-scale turbulent lifted hydrogen jet flame has been analysed to show that this flame has mixed mode combustion not only at the flame base but also in downstream locations. The mixed mode combustion is observed in instantaneous structures as in earlier studies and in averaged structure, in which the predominant mode is found to be premixed combustion with varying equivalence ratio. The nonpremixed combustion in the averaged structure is observed only in a narrow region at the edge of the jet shear layer. The analyses of flame stretch show large probability for negative flame stretch leading to negative surface averaged flame stretch. The displacement speed-curvature correlation is observed to be negative contributing to the negative flame stretch and partial premixing resulting from jet entrainment acts to reduce the negative correlation. The contribution of turbulent straining to the flame stretch is observed to be negative when the scalar gradient aligns with the most extensive principal strain rate. The physics behind the negative flame stretch resulting from turbulent straining is discussed and elucidated through a simple analysis of the flame surface density transport equation.

Keywords: Partially Premixed Flame, Flame Surface Density, Displacement Speed, Turbulent flame stretch, Lifted jet flame, Mixed mode combustion, 


\section{Introduction}

The occurrence of mixed mode combustion in many practical devices such as stratified charge direct injection engines and lean burn gas turbine combustor is common. In these devices, typically fuel at sufficiently high pressure is injected into the combustion chamber either intermittently or continuously resulting in a lifted flame. The detail of stabilisation mechanism is configuration dependent. However, the mechanisms of turbulence-flame interaction in the downstream part of the stabilisation region is expected to be device independent, especially in the continuous combustion systems. The combustion in these flames will likely to be in both premixed and nonpremixed modes.

Combustion models are required to close the mean reaction rate in ReynoldsAveraged-Navier-Stokes (RANS) calculations and filtered chemical reaction rate in Large Eddy Simulation (LES) of the above devices. The contribution of nonpremixed part to the mixed mode combustion can be included using an appropriate combustion model as has been done in an earlier study (Domingo et al., 2002). One popular approach to model premixed part is the flame surface density (FSD) based modelling (Pope, 1988, Candel and Poinsot, 1990; Trouvé and Poinsot, 1994; Vervisch et al., 1995). The balance equation for the FSD, $\Sigma$, which is defined as the flame surface area per unit volume, is written as (Veynante and Vervisch, 2002)

$$
\frac{\partial \Sigma}{\partial t}+\frac{\partial\left\langle u_{j}+S_{d} n_{j}\right\rangle_{s} \Sigma}{\partial x_{j}}=\langle\Phi\rangle_{s} \Sigma,
$$

using the usual nomenclature. In this equation the surface average or gradient weighted average of a quantity $Q$ is given by $\langle Q\rangle_{s}=\left(\left(\overline{Q|\nabla c|) \mid c=c^{*}}\right) /\left(\overline{|\nabla c| \mid c=c^{*}}\right)\right.$, where $c=c^{*}$ is a given iso-surface. The instantaneous flame stretch rate, $\Phi$, is defined as (Pope, 1988; Candel and Poinsot, 1990; Law, 2006)

$$
\begin{aligned}
\Phi & =\frac{1}{\delta A} \frac{\mathrm{d}(\delta A)}{\mathrm{d} t}=a_{T}+2 S_{d} K_{m} \\
& =\left(\delta_{i j}-n_{i} n_{j}\right) \frac{\partial u_{i}}{\partial x_{j}}+S_{d} \frac{\partial n_{i}}{\partial x_{i}}
\end{aligned}
$$

where $n_{i}$ is the component of flame normal unit vector, $\mathbf{n} \equiv-\nabla c /|\nabla c|$, in direction $i$. The flame normal unit vector is pointing towards the reactant side. The flame stretch consists of two components: the tangential strain rate, $a_{T}$, and a contribution from the joint behaviour of the flame surface displacement speed, $S_{d}$, and its mean curvature, $K_{m}$. It is to be noted that the above equations and various quantities involved in them are for a local differential area element in this study rather than for a continuous surface commonly followed while analysing turbulent premixed flames.

The instantaneous flame stretch can be positive or negative; a positive value implies that the flame surface area increases due to the combined effects of turbulence and flame propagation and negative stretch suggests that the flame surface is compressed resulting in the loss of flame area per unit volume. Earlier numerical (Rutland 
and Trouvé, 1993; Bray and Cant, 1991; Chen and Im, 1998), experimental (Nye et al., 1996: Renou et al., 1998) and modelling (Kostiuk and Bray, 1993) studies have demonstrated that there is $20-50 \%$ probability for the flame stretch to be negative. In the view of RANS methodology the average value of the flame stretch, $\langle\Phi\rangle_{s}$, is expected to be predominantly positive and many modelling methods have been proposed in the past with this view. A summary of these studies is provided by Veynante and Vervisch (2002) and Cant (2011).

It is well recognised that the scales involved in LES modelling are different from those involved in RANS. Thus, the contributions of the negative stretch can become important and must be considered appropriately in sub-grid scale (SGS) combustion models, as LES is becoming an attractive approach for combustion calculation because of its ability to capture unsteady phenomena in turbulent combustion. From Eq. (2) one observes that there will be three contributions to the filtered stretch rate; the first contribution comes from the filtered tangential strain rate part, the second contribution comes from the filtered curvature related term and the third contribution comes from the sub-grid scales. As Cant (2011) points out, it is convenient to combine the curvature term with the third, the flame propagation, term in the LHS of Eq. (1). Some models have been proposed in the past and can be found in Veynante and Vervisch (2002) and Cant (2011). The aim of this study is not to develop new or improved models but to provide plausible physical reasoning for the origin of negative flame stretch. The focus is on the mixed mode combustion because of its practical relevance and importance, specifically for gas turbine combustion. This is achieved by analysing direct numerical simulation (DNS) data of a laboratory scale lifted turbulent jet flame (Mizobuchi et al., 2002, 2005). The instantaneous and intermittent mixed mode nature of this lifted flame has been demonstrated in earlier studies (Mizobuchi et al., 2002, 2005). Here, it is shown that the mixed mode combustion occurs even in an averaged sense.

Many DNS (Swaminathan and Grout, 2006; Chakraborty and Swaminathan, 2007a, Kim and Pitsch, 2007; Mura et al., 2008, 2009; Chakraborty et al., 2009; Richardson et al. 2010: Chakraborty et al. 2010) and experimental (Hartung et al., 2008; Steinberg et al. 2012) studies of premixed combustion and stratified combustion (Malkeson and Chakraborty, 2011) have demonstrated that the reactive scalar gradient vector aligns with the most extensive principal component of the turbulent strain rate when the local heat release is strong. This is in contrast to the passive scalar physics where the scalar gradient is known to align with the most compressive component. This change in the alignment of scalar gradient with the principal strain rate results in a decrease of the scalar gradient in turbulent premixed flames. The rotation of principal strain planes due to dilatational effects might also be a possible cause for the decrease of scalar gradient when the heat release is moderate (Gonzalez and Paranthoën, 2011). It is known (Swaminathan and Grout, 2006) that this alignment is directly related to the tangential strain rate part of the flame stretch in Eq. (2) and thus the negative stretch rate can result from a change in the scalar gradient alignment. The questions that are of interest to this study are, viz., (i) Is this alignment change solely responsible for the 
negative stretch rate or the curvature term in Eq. (2) plays a role as well? and (ii) What are their relative roles? The answers will have implications for SGS modelling of the flame stretch.

As it is clear from Eq. (2), the displacement speed, $S_{d}$, magnitude must be taken into consideration while analysing the flame stretch and its components. The behaviour of $S_{d}$ in perfectly premixed flame has been studied extensively (Echekki and Chen, 1996; Chen and Im, 1998, 2000; Hawkes and Chen, 2005; Chakarborty and Cant, 2005). However, it has received limited attention in partially premixed and stratified flames (Helié and Trouvé, 1998; Bray et al., 2005; Malkeson and Chakraborty, 2010). The dependence of progress variable on mixture fraction yields additional contributions, which is written as (Bray et al. 2005)

$$
S_{d}^{*}=S_{d}^{0}+\frac{1}{\rho|\nabla c|} \frac{1}{\partial Y_{i} / \partial c}\left[2 \rho N_{c z} \frac{\partial^{2} Y_{i}}{\partial Z \partial c}+\rho N_{Z Z} \frac{\partial^{2} Y_{i}}{\partial Z^{2}}+\rho N_{c c} \frac{\partial^{2} Y_{i}}{\partial c^{2}}\right],
$$

where $Y_{i}$ is the mass fraction of species $i$ and

$$
S_{d}^{0}=\frac{1}{\rho|\nabla c|}\left[\dot{\omega}_{c}+\frac{\partial}{\partial x_{i}}\left(\rho D \frac{\partial c}{\partial x_{i}}\right)\right]
$$

containing contributions from the chemical reaction rate and molecular diffusion as for the perfectly premixed flames. For the sake of clarity, it is noted that the displacement speeds defined in Eqs. (3) and (4) are not density weighted because there may be neither fully burnt nor unburnt mixtures across the flame surface in partially premixed combustion. Also, the flame surface may not be simply connected in partially premixed combustion because the reactant mixture is spatially and temporally inhomogeneous. The additional contributions for partially premixed flames come from three scalar dissipation rates defined as $N_{Z Z}=\rho D(\nabla Z \cdot \nabla Z), N_{c z}=\rho D(\nabla c \cdot \nabla Z)$ and $N_{c c}=\rho D(\nabla c \cdot \nabla c)$, where $D$ is the molecular diffusivity which is taken to be equal for the progress variable and mixture fraction. This is acceptable since Eq. (3) is strictly valid when the molecular diffusivities of all the species are equal (Bray et al., 2005). For non-equal difficulties Eq. (3) becomes

$$
\hat{S}_{d}^{*}=S_{d}^{*}-\frac{\nabla \cdot\left[\rho\left(D-D_{i}\right) \nabla c\right]}{\rho|\nabla c|}-\frac{\nabla \cdot\left[\rho\left(D-D_{i}\right) \nabla Z\right]}{\rho|\nabla c|} \frac{\left(\partial Y_{i} / \partial Z\right)}{\left(\partial Y_{i} / \partial c\right)} .
$$

These differential diffusion terms may be negligible in high Reynolds number flow and are neglected in this study.

Malkeson and Chakraborty (2010) observed that the additional contributions from the dissipation rates were small while analysing their DNS data of turbulent planar stratified flames with initial mixture inhomogenity introduced in a direction normal to the mean flame propagation direction. The combustion kinetics of hydrocarbon flames was simulated using a single step reaction with activation energy and heat release depending on local equivalence ratio. The effect of mixture stratification in transverse direction has also been studied in turbulent (Anselmo-Filho et al., 2009) and laminar 
(Dold, 1989) flames, but its influence on the additional contributions in Eq. (3) has not been addressed yet. It has been noted in the past studies that the mixture stratification in the transverse direction yields triple flames (Kioni et al., 1993; Ruetsch et al., 1995; Plessing et al., 1998). Also, the behaviour and contributions of these additional terms in the presence of significant flame curvature, complex chemical kinetics, differential diffusion effects and a wide range of local flow and mixture conditions are not investigated yet. Furthermore, their contributions to the flame stretch are yet to be studied. The DNS dataset of a laboratory scale lifted turbulent hydrogen flame simulated with detailed chemistry and transport offers a good opportunity to address these effects. Thus, the specific objectives are

- To study turbulence-scalar interaction and its influence on flame stretch in a turbulent lifted jet flame depicting mixed mode combustion in its averaged structure,

- To study the influence of partial premixing resulting from the jet entrainment on the displacement speed-curvature correlation, and

- To find physical reasons for negative values of surface averaged flame stretch, if it exists, in this mixed mode combustion.

This paper is organised as follows. The next section provides pertinent detail on the DNS data and its processing briefly. Results are discussed in section III and the conclusions are summarised in section IV.

\section{DNS and data processing}

Details of the DNS data used in this study can be found in Mizobuchi et al. (2002, 2005) and thus only a brief discussion on attributes relevant for this study is given here. An experimentally investigated (Cheng et al., 1992, 2007) turbulent lifted flame established above a hydrogen jet issuing from a round nozzle into quiescent air was simulated using direct numerical simulation methodology. The jet Mach number is 0.54 and its Reynolds number based on the nozzle diameter is 13,600. A schematic of this flow configuration is shown in Fig. 1 a (to be discussed fully later) along with a typical instantaneous temperature field and the stoichiometric mixture fraction contour. The streamwise, $y$, and cross-stream distances are normalised by the nozzle diameter, $D=2 \mathrm{~mm}$. The size of the computational domain was $\pm 12.5 \mathrm{D}$ in the cross-stream directions and $-2 D$ to $20 D$ in the streamwise direction. The domain is discretised using a non-uniform grid with a total of 200 million grid points. The DNS was considered (Mizobuchi et al., 2002, 2005; Ruan et al., 2012) to be well-resolved, with a uniform grid spacing of $0.05 \mathrm{~mm}$ within $\pm 5 D \times 14.75 D \times \pm 5 D$. This grid spacing is about 2.5 times the Kolmogorov length scale close to ignition point in the experiment. The ratio between the stoichiometric laminar premixed flame thermal thickness and 
the DNS mesh resolution is about 10. The stoichiometric planar unstrained laminar premixed flame solution computed using the DNS grid is compared to the CHEMKIN solution with adaptive gridding in Fig. 2. This suggests that the flame front structure is captured well and the DNS resolution is adequate to address the objectives of this study. Furthermore, this numerical resolution was verified to be sufficient to study scalar and velocity gradients related statistics by Ruan et al. (2012). This DNS employed a chemical kinetics mechanism involving 9 species (including nitrogen as an inert species) and 17 reactions (Westbrook, 1982). The transport properties depend on temperature and local species concentration. The flame lift-off height is about 5.7D from the nozzle exit in the DNS while it was $7 D$ in the experiments of Cheng et al. (1992, 2007). The comparison of flame brush structure, in terms of mean species mass fractions and temperature, obtained from the DNS to that measured was shown to be good by Ruan et al. (2012).

Two data processing methods have been used in this study. The first one is similar to a RANS-type of averaging detailed in Ruan et al. (2012) in order to investigate mean flow and flame quantities. The scalar gradients are first calculated by a central difference scheme. The statistics in a given cross section at streamwise location is constructed by splitting the cross section into a number of concentric rings as shown in Fig. $1 \mathrm{~b}$ and the symbols $\Delta, \mathbf{B} \mathbf{1}$ and $\mathbf{C 1}$ will be explained later. The radial distance $R=\sqrt{x^{2}+z^{2}}$ is measured from the jet centre-line $(R=0)$. All the sample points in a particular ring of width $d r$ are averaged, as has been done by Ruan et al. (2012) to obtain a mean value as

$$
\bar{Q}(R, y)=\frac{1}{N_{t} N} \sum_{t_{i}=1}^{N_{t}} \sum^{N} Q\left(x, z, t_{i} ; y\right),
$$

where $Q$ is the quantity of interest, $N_{t}(=146)$ is the number of sampling over a period of $0.09 \mathrm{~ms}$ and $N$ is the total number of data points in a particular ring of width $d r$ for one instant. In this study the time and spatial averaging are combined to increase the sample size for statistical accuracy since the flow and flame have reached statistically stationary state. This has been verified by Ruan et al. (2012) by increasing the sampling period by nearly 3 times and reconstructing appropriate statistics. Sensitivity of these statistics to the ring width $d r$ has also been tested and it was observed to be small. Thus the results reported in this study are for $d r=2 d x$ unless specified otherwise. Statistical convergence of the results presented in this study has also been verified by increasing the sample size and sampling duration (Ruan et al., 2012). Although the sample size in this ring averaging method varies with radius, it increases very quickly; the sample contains more than 14,000 data for $R / D=0.2$ which is expected to provide meaningful statistics. The region of interest where significant reactions occur are located mostly at $R / D>1.0$ and thus the sample size is sufficiently large. For the centreline values, 969 DNS data samples over $0.5 \mathrm{~ms}$ are ensemble averaged to obtain the required statistics. The above method is also used appropriately to obtain Favre averaged quantities.

The second method is similar to LES-type filtering using a box filter in physical space. In this method, one single ring is split into 72 evenly distributed arc sections 


\begin{tabular}{l|cccccc}
\hline Position & $R / D$ & $\Delta$ & $\delta_{L}^{0}$ & $\Lambda$ & $\eta$ & $\tau_{t}$ \\
\hline B1 & 3 & 0.53 & 0.47 & 1.02 & 0.16 & 0.23 \\
C1 & 4 & 0.68 & 0.44 & 0.71 & 0.14 & 0.17 \\
\hline
\end{tabular}

Table 1: Turbulence integral, $\Lambda$, and Kolmogorov, $\eta$, length scales, integral time scale, $\tau_{t}$, and planar laminar premixed flame thermal thicknesses, $\delta_{L}^{0}$, at positions $\mathbf{B 1}$ and $\mathbf{C 1}$. Length scales are in $\mathrm{mm}$ and time scale is in $\mathrm{ms}$.

and this is expected to reduce the sample size. Thus, to maintain the statistical accuracy and convergence the ring width is increased to $6 d x$ and samples in 5 consecutive time steps are combined to get flame surface density and surface averaged quantities required for flame stretch calculation. The typical arc width, $\Delta$, is marked in Fig. 10 for two arbitrary locations, $\mathbf{B} \mathbf{1}$ and $\mathbf{C 1}$, chosen for analysis. These two locations are not at the same streamwise, $y$, distance from the nozzle exit; $\mathbf{B} 1$ is on the plane B-B marked in Fig. 11 and $\mathbf{C 1}$ is on the plane C-C. These two locations are also marked in Fig. 3, to be discussed later, as black circles to indicate their relevance with respect to the averaged temperature and $\mathrm{OH}$ mass fraction. The filter widths, radial positions and other relevant scales at these two locations are given in Table 1 . The laminar flame thermal thicknesses are calculated using a hydrogen-air mixture with a value of mixture fraction, $Z$, equal to the Favre averaged values at these locations. The turbulence scales are estimated using the Favre averaged turbulent kinetic energy and its dissipation rate at these locations. There is no significant change in the turbulence scales at these two locations due to the contributions from shear generated turbulence. Ideally one would like to have $\Delta \leq \Lambda$, which is satisfied at $\mathbf{C 1}$. The conditions at $\mathbf{B 1}$ gives $\Delta / \delta_{L}^{0} \approx 1.1, \delta_{L}^{0} / \eta \approx 2.8$ and $\Lambda / \delta_{L}^{0} \approx 2.2$. The statistics presented in section 3.5 for the locations $\mathbf{B} 1$ and $\mathbf{C 1}$ are constructed using the second method of data processing described above.

In this study, the mixture fraction is calculated using (Bilger, 1988)

$$
Z=\frac{\xi_{\mathrm{H}} / W_{\mathrm{H}_{2}}+2\left(Y_{\mathrm{O}_{2}, \text { air }}-\xi_{\mathrm{O}}\right) / W_{\mathrm{O}_{2}}}{1 / W_{\mathrm{H}_{2}}+2 Y_{\mathrm{O}_{2} \text {,air }} / W_{\mathrm{O}_{2}}},
$$

where the molecular weights are $W_{\mathrm{O}_{2}}=32$ and $W_{\mathrm{H}_{2}}=2, \xi_{i}$ is the mass fraction of element $i$ and $Y_{\mathrm{O}_{2} \text {,air }}=0.244$ is the mass fraction of $\mathrm{O}_{2}$ in air with $22 \% \mathrm{O}_{2}$ and $78 \%$ $\mathrm{N}_{2}$ by volume. This definition gives $Z_{s t} \approx 0.03$ for the stoichiometric mixture fraction. The mass diffusivity, $D$, of $Z$ is calculated with a mass weighted individual species diffusivity $D_{i}$ as $D=\sum Y_{i} D_{i}$.

The progress variable $c$ depends on $Z$ in partially premixed flames and is defined in this paper using the product mass fraction $Y_{\mathrm{H}_{2} \mathrm{O}}$ as

$$
c(Z)=\frac{Y_{\mathrm{H}_{2} \mathrm{O}}}{Y_{\mathrm{H}_{2} \mathrm{O}}(Z)},
$$


where $Y_{\mathrm{H}_{2} \mathrm{O}}^{E q}(Z)$ is the equilibrium value corresponding to the local mixture fraction $Z$. The mass diffusivity of this $c$ is equal to $D_{\mathrm{H}_{2} \mathrm{O}}$. The progress variable can also be defined using hydrogen mass fraction, which was observed to be similar to that based on $\mathrm{H}_{2} \mathrm{O}$ as has been shown by Ruan et al. (2012) for this turbulent flame.

\section{Results and Discussion}

In this section the global mean flame and flow quantities are discussed first followed by an analysis of the scalar-turbulence interaction in the lifted flame. The contributions of additional terms arising due to partial premixing in displacement speed expression is investigated and then the correlation of the displacement speed with flame curvature is examined. These behaviours are then linked to the negative values of surface averaged flame stretch observed in this flame. After presenting these statistics in the RANS view, temporal evolution of local flame surface density, tangential strain rate, curvature related quantities and the flame stretch are probed at the two arbitrarily chosen locations, B1 and $\mathbf{C 1}$ to shed light on how the local flame stretch is influenced by the scalar-turbulence interaction and the displacement speed-curvature correlations.

\subsection{Flame brush attributes}

Figure 3 shows the mean temperature and $\mathrm{OH}$ mass fraction fields along with iso-lines of $\bar{Z}$. The stoichiometric mixture fraction line is shown by the solid line. The mean values are obtained using Eq. (6). The peak mean $\mathrm{OH}$ values corresponds to the peak mean temperatures as one would expect and these peak locations are in the neighbourhood of the stoichiometric mixture fraction. Also, these averaged fields show that the flame lift-off height is about 5.7D. Although these averaged temperature, $\mathrm{OH}$ and mixture fraction fields suggests a diffusion flame type structure (high $\bar{T}, \bar{Y}_{\mathrm{OH}}$ near $\bar{Z}_{\text {st }}$ ) in a mean sense, a detailed analysis of instantaneous reaction rate and structure of reactions zones suggests a predominantly premixed type combustion with varying equivalence ratio and occasional diffusion flame island occurring at downstream locations (Mizobuchi et al., 2002, 2005; Ruan et al., 2012).

The presence premixed and nonpremixed modes of fuel consumption can be identified by plotting the fuel consumption rate and the flame index together. The flame index defined (Yamashita et al., 1996) as

$$
\mathrm{FI}=\nabla Y_{\mathrm{H}_{2}} \cdot \nabla Y_{\mathrm{O}_{2}}
$$

is negative for non-premixed mode since the hydrogen and oxygen are diffusing from the opposite directions. This index is positive for premixed mode because the fuel and oxidiser gradients are aligned with one another. Hence, the non-premixed and premixed regions can be demarcated using the FI $=0$ contour. It must be noted that FI can be zero in regions with no combustion and thus its use in conjunction with reaction rate is more useful. Such an analysis has been performed in earlier studies (Mizobuchi 
et al., 2002, 2005) using this DNS data suggesting the presence of inner cone of rich premixed flame and outer diffusion flame islands in instantaneous images. Figures $4 \mathrm{a}$ to 4 c depicting color maps of the instantaneous fuel consumption rate and, FI $=0$ and $Z_{s t}$ contours show the above arrangement of rich premixed and diffusion flames. The FI contour is shown only for the region $2 D \leq|x| \leq 7 D$ in Fig. $4 a$ and $4 \mathrm{p}$ for clarity. If one plots this contour for the entire range of $x$ then they will be very crowded near the central region and will be similar to that shown by Yoo et al. (2009) in their figure 11. Also, one observes that the diffusion mode of fuel consumption occurs in isolated islands on the outer side of the jet in downstream region and this extends to $y=5.5 D$ in instantaneous images shown in Fig. $4 \mathrm{a}$ and $4 \mathrm{~b}$.

Although the above arrangement of premixed and diffusion flames in the lifted flame has been reported earlier (Mizobuchi et al., 2002, 2005), this result is shown here only for the sake of completeness. These premixed and diffusion flames are observed to be highly intermittent as noted by Mizobuchi et al. (2002, 2005). Hence, the averaged image may portray a different picture and this averaged image is shown in Fig. $4 \mathrm{~d}$. The triple flame feature present at the flame-base in instantaneous snap-shots are not seen in the averaged image and the predominant fuel consumption is through rich premixed combustion with varying equivalence ratio. There is also lean premixed combustion, which is signified by $0.006 \leq \overline{\dot{\omega}}_{H 2} \leq 0.013$ for $\bar{Z}<\bar{Z}_{\text {st }}$ and $\overline{\mathrm{FI}}>0$ in the averaged image shown in Fig. $4 \mathrm{~d}$. The predominant rich premixed mode at the base is preceded by a moderate reaction zone with $\bar{Z}>\bar{Z}_{\text {st }}$ and $\overline{\mathrm{FI}}<0$. The negative value of $\overline{\mathrm{FI}}$ suggests a nonpremixed combustion but the mixture fraction contour suggests a contrary. Thus, this particular reactive region having an area weighted mean rate of about $15 \%$ of the maximum reaction rate seen in Fig. $4 \mathrm{~b}$ results from the highly intermittent diffusion and rich premixed branches of the triple flame structure at the flame-based observed in Figs. $4 \mathrm{a}$ and $4 \mathrm{~b}$. From these results one notes that the premixed and non-premixed modes coexist in the flame brush for $y \geq 10 D$ and the predominant fuel consumption is through the premixed combustion with varying equivalence ratio. The presence of nonpremixed mode at the base of the flame brush is unclear and the premixed mode is observed to be dominant. Hence, it is clear that this lifted flame involves mixed mode combustion even in an averaged sense.

The three axial positions chosen for further analysis of this flame with mixed mode combustion are marked in Figs. 1 and 3. The position A-A is close to the flame-base and the location B-B is selected because experimentally measured flame brush structure is available for validation as has been discussed by Ruan et al.(2012). The location $\mathrm{C}-\mathrm{C}$ is the most downstream position, where the grid is uniform and occasional diffusion flame islands are observed in the instantaneous fields (Mizobuchi et al., 2005, Ruan et al., 2012) (also see Fig. 4). The radial variation of $\widetilde{Z}$ and $\widetilde{c}$ at these three axial positions is shown in Fig. 5. The centreline value of $\widetilde{Z}$ decreases with downstream position as one would expect. The Favre mean progress variable increases from the centreline and then drops gradually after reaching a peak value. The increase is due to the formation of $\mathrm{H}_{2} \mathrm{O}$ in the flame brush and its diffusion towards the jet centre. Thus, the centreline value of $\widetilde{c}$ increases as one moves in the downstream direction. The drop 
in $\widetilde{c}$ after reaching a peak value in the radial direction is due to the mixing of combustion product with the quiescent air. One can also observe that the peak value of $\widetilde{c}$ shifts radially outward from the position A-A to C-C. The variation of $\widetilde{c}$ in Fig. 5 also suggests that the flame brush thickness increases in the downstream direction. All of these general behaviours are as expected for the flow and flame configuration considered in the DNS.

The stoichiometric mixture fraction value is $Z_{s t}=0.03$ and, the lean and rich flammability limits $(\overline{\mathrm{Law}}, 2006)$ are respectively $Z_{l}=0.005$ and $Z_{r}=0.175$ for the hydrogen-air mixture used in the DNS. If the combustion occurs predominantly in non-premixed mode then the peak $\widetilde{c}$ would be roughly around $\widetilde{Z}=Z_{s t}$, which is not one observes in Fig. 5. The peak location is on the lean side for the position A-A suggesting a lean premixed combustion mode. For the downstream locations the peak is broad and covers a significant portion of the flammability range suggesting partially premixed mode - rich, stoichiometric and lean premixed with non-premixed combustion. These observations are consistent with the insights obtained from Fig. 4 and lends further support to call this flame as partially premixed flame. The rich partial premixing in the downstream of the flame-base is caused by the entrainment of surrounding air and the leakage of oxygen through the reaction zones in the flame stabilisation region (Mizobuchi et al., 2002, 2005). The diffusion flame islands in the downstream regions are formed due to the radial diffusion of hydrogen from the inner rich premixed cone and the radially entrained air. The leakage of oxygen in the radially entrained air through the diffusion flame and those coming through the flame-base establishes premixed flames of varying equivalence ratio at downstream regions. The oxygen leakage through a laminar $\mathrm{H}_{2}$-air diffusion flame is verified using CHEMKIN. The oxygen leakage in hydrocarbon flames is well known (Smooke and Givangoli, 1991) and hence it is likely that the mixed mode combustion as noted above can be seen for hydrocarbons as well. Some evidence for this can be seen in figure 11 of Luo et al. (2012) who investigated autoigniting ethylene jet in a hot coflow. Thus, the occurrence of mixed mode combustion in a lifted flame is not fuel specific and this generic feature is one of the important characteristics of turbulent lifted flame.

\subsection{Turbulence-scalar interaction}

The tangential strain rate $a_{T}$ in Eq. (2) has been shown to relate closely to the turbulencescalar interaction process, which is signified by the inner scalar product of scalar gradient vector and turbulent strain tensor (Swaminathan and Grout, 2006). This is written as

$$
T_{3}=\underbrace{-2 \overline{\rho D} \frac{\partial \widetilde{c}}{\partial x_{i}}\left(\frac{\partial c^{\prime \prime}}{\partial x_{j}} \frac{\partial u_{i}^{\prime \prime}}{\partial x_{j}}\right)}_{T_{31}} \underbrace{-2 \rho D \frac{\partial c^{\prime \prime}}{\partial x_{j}} \frac{\partial u_{i}^{\prime \prime}}{\partial x_{j}} \frac{\partial c^{\prime \prime}}{\partial x_{i}}}_{T_{32}} \underbrace{-2 \rho D \frac{\partial c^{\prime \prime}}{\partial x_{i}} \frac{\partial c^{\prime \prime}}{\partial x_{j}} \frac{\partial \tilde{u}_{i}}{\partial x_{j}}}_{T_{33}} .
$$

The radial variation of these three terms, normalised using the stoichiometric unburnt mixture density, $\rho_{u, s t}$, and its flame time $t_{f}=\delta_{L, s t}^{0} / S_{L, s t}^{0}$, is shown in Fig. 6 for the three 
axial locations. The mean scalar and velocity gradients terms, $T_{31}$ and $T_{33}$ respectively, are small and the dominant contribution comes from the triple correlation $T_{32}$. This quantity shown in Fig. 6 is little noisy because of the sample size available for averaging. However, increasing the sample size by increasing the ring width does not unduly change the results, specifically the positive and negative contributions. Thus, these contributions are physical and not due to insufficient sample size. The $T_{32}$ term is positive in general implying the generation of the iso-scalar surface area by turbulence. The negative values imply that $T_{32}$ dissipates the surface area as has been observed in previous DNS studies of turbulent premixed (Swaminathan and Grout, 2006, Chakraborty and Swaminathan, 2007a $b$; Kim and Pitsch, 2007) and stratified flames (Malkeson and Chakraborty, 2011). However, the negative values are seen for narrow regions in Fig. 6. This dissipation is because the heat release effects are stronger than the turbulence processes (see Fig. 3 showing high temperature in these regions).

The RMS (root-mean-square) value of turbulent velocity fluctuations may be used as an indicator for the local turbulence level and the laminar flame speed may be compared to this RMS value to understand relative roles of heat release and turbulence processes. One must also bear in mind that the local flame speed can vary significantly in partially premixed flames since the local mixture fraction value can change. Figure 7 shows the radial variation of the RMS velocity normalised by the laminar flame speed for the local Favre mean mixture fraction value. The RMS velocity is calculated using $U_{r m s}^{2}=2 \widetilde{k} / 3$, where $\widetilde{k}$ is the Favre averaged turbulent kinetic energy at the given location. By comparing Figs. 6 and 7 it is clear that the RMS velocity and laminar flame speed are of the same order, more precisely the normalised RMS velocity is smaller than five, in the regions of negatives $T_{32}$, specifically for $R / D \approx 3$ at the location $\mathrm{B}-\mathrm{B}$ and $R / D \approx 4$ at the location $\mathrm{C}$-C. Although $U_{r m s}^{+}$is about three at $R / D \approx 2$ for the location A-A, there is no strong heat release at this location as one observes in Figs. 1 and 3. Since it is the flame-base, the turbulence effects dominate resulting in the generation of iso-scalar surface area.

To further understand $T_{32}$, it is written using the eigenvalue decomposition as (Swaminathan and Grout, 2006; Chakraborty and Swaminathan, 2007a b; Kim and Pitsch, 2007; Malkeson and Chakraborty, 2011)

$$
T_{32}=-2 \rho D\left(\frac{\partial c^{\prime \prime}}{\partial x_{i}} \frac{\partial c^{\prime \prime}}{\partial x_{i}}\right)\left(\alpha \cos ^{2} \theta_{\alpha}+\beta \cos ^{2} \theta_{\beta}+\gamma \cos ^{2} \theta_{\gamma}\right),
$$

where $\alpha>\beta>\gamma$ are the eigenvalue of the symmetric turbulent strain tensor $e_{i j}^{\prime \prime}=$ $0.5\left(\partial u_{i}^{\prime \prime} / \partial x_{j}+\partial u_{j}^{\prime \prime} / \partial x_{i}\right)$, with $\alpha$ as the most extensive and $\gamma$ as the most compressive principal strains. The angles between the scalar gradient vector and the eigenvector $i$ is denoted by $\theta_{i}$. The sign of $T_{32}$ is determined by the predominant alignment of the scalar gradient with the principal direction, which determines the characteristics of the scalar-turbulence interaction. The $T_{32}$ will be positive or negative if the predominant alignment is with the $\gamma$ or $\alpha$ strain respectively.

The pdfs of direction cosines are shown in Fig. 8 for $\alpha$ and $\gamma$ strains at various radial and axial positions in the lifted flame investigated here. In general, alignment 
with the most compressive strain is observed for almost all the locations shown except for $R / D=3$ at $\mathrm{B}-\mathrm{B}$ and $R / D=4$ at $\mathrm{C}-\mathrm{C}$. An alignment with the most extensive strain is observed for $R / D=3$ at the location B-B where $T_{32}<0$ as noted earlier. However, there is no clear preferential alignment at $R / D=4$ for the location $\mathrm{C}-\mathrm{C}$ although negative $T_{32}$ was observed for this location. A careful and thorough analysis of the DNS data at this location indicates that the flame fronts are intermittent (see Fig. 4) and thus this orientation statistics may be biased. However, one must note that the sign of $T_{32}$ is also influenced by the relative magnitude of $\alpha$ and $\gamma$ in Eq. (11) and it is apparent from the behaviour of $T_{32}$ in Fig. 6 that the magnitude of $\alpha$ is larger than $\gamma$ at $R / D=4$ for the location C-C. This position is marked as $\mathbf{C 1}$ in Fig. 3 and we shall investigate, in section 3.5 , the temporal variation of flame stretch, flame surface density, tangential strain rate and curvature related term at this position for a closer understanding.

In order to address the objective on the negative flame stretch, one must also study the curvature related term in Eq. (2), which involves the iso-surface displacement speed. As noted earlier, the role of additional contributions to the displacement speed need to be investigated first before studying the curvature-displacement speed correlation in partially premixed flames.

\subsection{Partial premixing influence on displacement speed}

Bray et al. (2005) discussed the additional contributions due to partial premixing and wrote the displacement speed in Eq. (3) as

$$
S_{d}^{*}=S_{d}^{0}+A^{*}+B^{*},
$$

where $S_{d}^{0}$ is given by Eq. (4). Using the definition of $c$ in Eq. (8) one gets

$$
\begin{aligned}
A^{*} & =\frac{2 N_{c z}}{|\nabla c| Y_{H 2 O}^{E q}(Z)} \frac{\mathrm{d} Y_{H 2 O}^{E q}(Z)}{\mathrm{d} Z}, \\
B^{*} & =\frac{c N_{Z Z}}{|\nabla c| Y_{H 2 O}^{E q}(Z)} \frac{\mathrm{d}^{2} Y_{H 2 O}^{E q}(Z)}{\mathrm{d} Z^{2}},
\end{aligned}
$$

for $Y_{i}=Y_{\mathrm{H}_{2} \mathrm{O}}$ iso-surface. The additional contributions include chemical kinetic effect and the turbulent mixing at small scales. The chemical kinetic effects come through the first and second derivatives in the mixture fraction space and the small-scale turbulent mixing is felt through the scalar dissipation rates, $N_{Z Z}$ and $N_{c Z}$. The chemical

kinetic terms are shown in Fig. 9, by plotting the variation of $\Psi=Y_{\mathrm{H}_{2} \mathrm{O}}^{E q}, \Psi^{\prime}=\mathrm{d} \Psi / \mathrm{d} Z$ and $\Psi^{\prime \prime}$ with $Z$. Note that the values are scaled appropriately to fit within the range shown in this figure. These values are obtained by performing equilibrium calculations by allowing the species involved in the chemical kinetic mechanism used in the DNS to be present in the equilibrium mixture. The burnt side values of these species 
in freely propagating adiabatic planar laminar flames are also used to verify the equilibrium values. As one would expect, large changes in $\Psi^{\prime}$ and $\Psi^{\prime \prime}$ are close to the stoichiometric value, $Z_{s t}=0.03$. The first derivative is positive for $Z<0.04$, zero for $Z=0.042$ and approaches a value of about -0.275 for $Z>0.07$. The second derivative is negative reaching a peak at $Z_{s t}$ and approaching zero for $Z<0.015$ and $Z>0.05$ and thus the additional contribution of $B^{*}$ will be significant only in this narrow range around the stoichiometry. Also, the second derivative is nearly 100 times larger than the first derivative and thus the contribution of $B^{*}$ is expected to be larger than $A^{*}$. The contribution of $A^{*}$ is expected only for $Z<0.042$ and this will be compounded by the cross dissipation rate behaviour and its magnitude. Ruan et al. (2012) have shown that the cross dissipation rate is an order of magnitude smaller than the mixture fraction dissipation rate and thus the combined contributions of chemical kinetics and mixing through $A^{*}$ is expected to be negligible.

The above observations have been confirmed by calculating the gradient weighted averages of the displacement speed, $S_{d}^{*}$, and its components, and the results are shown in Fig. 10 as the radial variation of these quantities for the three axial locations, A-A, B-B and C-C. In general $\left\langle S_{d}^{0}\right\rangle_{s}$ is positive and contributes predominantly to the total displacement speed, $S_{d}^{*}$, which is also positive. The contributions of $A^{*}$ can be both positive and negative and it is an order of magnitude smaller than $S_{d}^{0}$ for the reasons noted above. Thus, it can be neglected from further consideration. The contribution of $B^{*}$ is negative and mainly around the stoichiometry. Its relative importance increases at downstream locations; a contribution of about $40 \%$ can be observed for the location C-C, which is consistent with previous DNS studies (Helié and Trouvé, 1998). The increased contribution at downstream location is because of the presence of diffusion flame islands and the form of $B^{*}$ in Eq. (14), the second derivative in the mixture fraction space multiplied by the mixture fraction dissipation rate, clearly notes that this contribution is from nonpremixed combustion mode. However, it is worth noting that Bray et al. (2005) did not distinguish premixed or nonpremixed combustion modes from one another while developing Eqs. (3) to (14). Although the displacement speed concept for a fully non-premixed combustion may be less relevant, Eq. (14) represents the contribution of non-premixed mode to the instantaneous reaction rate $\dot{\omega}_{c}^{*}=\rho|\nabla c| S_{d}^{*}$ of the progress variable $c$ in the partially premixed combustion. This view helps one to develop a simple modelling framework for mixed mode combustion.

The effects of partial premixing is seen only near the mean stoichiometric isosurface as suggested by the results in Figs. 9 and 10 . The relative importance of these effects is influenced by the extent of partial premixing, which is typically quantified by the ratio of the RMS of mixture fraction fluctuation to its mean value, $Z_{r m s} / \widetilde{Z}$, the amount of heat release and turbulence intensity. The value of the heat release parameter is about 7 and stratification level is typically larger in the current DNS than in many previous studies; for example, $Z_{r m s} / \bar{Z} \approx 0.6$ and $U_{r m s}^{+} \approx 3.0$ at $R / D=2.75$ for the position $\mathrm{B}-\mathrm{B}$, and, $Z_{r m s} / \widetilde{Z} \approx 0.4$ and $U_{r m s}^{+} \approx 3.5$ at $R / D=3.75$ for the position C-C. 


\subsection{Displacement speed-curvature correlation}

The effects of partial premixing has been shown to decrease the total displacement speed in general for this flame (see Fig. 10p. Now, we like to examine its effect on $S_{d}^{*}$-curvature correlation since the flame stretch involves this as shown by Eq. (2). The pdf of curvature normalised using the thermal thickness of a planar unstrained laminar flame with the mixture fraction value equal to $\widetilde{Z}$ at a given position, ie., $K_{m}^{+}=K_{m} \delta_{L}^{0}(\widetilde{Z})$, is shown in Fig. 11 for several radial and axial positions. Since the position A-A is close to the base, the curvature pdf at this location is shown only for comparison. The pdfs show long positive or negative tail for some locations. The positive tail is seen near the flame-base and the negative tail is observed for a downstream location, which are purely due to the flame geometry. Nevertheless the mean curvature is close to zero in general as has been observed in many earlier studies.

The joint pdf of the curvature and the displacement speed, $P\left(K_{m}^{+}, S_{d}^{*} / S_{L}^{0}(\widetilde{Z})\right)$, is shown for few radial and axial locations in Fig. 12 . The displacement speed is normalised using the laminar flame speed $S_{L}^{0}$ corresponding to the local $\widetilde{Z}$ for a given position. Since the interest is to study the negative flame stretch, the locations for these joint pdfs are chosen to be inside the flame brush so that the laminar flame speed is non-zero, rather the Favre averaged mixture fraction value is well within the flammability limits of the hydrogen-air mixture (cf. Fig. 5). Also, the mean values of $K_{m}^{+}$ and $S_{d}^{*} / S_{L}^{0}$ are marked using a solid circle to help us to understand the change in the correlation easily. The numerical values for these means are also given in this figure. The correlation coefficients calculated using the joint pdfs shown in Fig. 12 are $-0.22,-0.04,-0.32$ and -0.37 respectively for $R / D=2$ and 3 at $\mathrm{B}-\mathrm{B}$, and $R / D=2$ and 3.75 at C-C locations. It is apparent that this correlation is negative even for this partially premixed flame. This negative correlation is consistent with previous studies on turbulent premixed (Gran et al., 1996; Chen and Im, 1998, 2000; Hawkes and Chen, 2005: Chakarborty and Cant, 2005) and stratified (Malkeson and Chakraborty, 2010) flames. The instantaneous flame surface with positive curvature can have negative displacement speed as noted by Gran et al. (1996) which is also seen in this flame. It is worth noting that Gran et al. (1996) considered premixed combustion of stoichiometric methane-air mixture while this study considers mixed mode combustion in a turbulent lifted hydrogen jet flame.

The $S_{d}^{*}-K_{m}$ correlation becomes more negative as one moves radially outwards for a given axial location, except for the flame-base (A-A location). This change is signified by the shift of the mean $S_{d}^{*}$ value away from the value of $S_{d}^{*}$ for which the pdf peaks. This is because of increased chemical activity resulting in increased reaction rate as indicated by the mean temperature and $\mathrm{OH}$ mass fraction fields in Fig. 3. To elucidate the effect of partial premixing on this correlation, the joint pdf, $P\left(K_{m}^{+}, S_{d}^{0} / S_{L}^{0}(\widetilde{Z})\right)$, after excluding the additional contributions from the partial premixing is shown in Fig. 13 for $R / D=3.75$ at the position C-C. This particular position is chosen for this because of the significant contribution of $B^{*}$ to the total displacement speed as shown in Fig.10. The overall pattern of the joint pdf in Fig. 13 is very similar to that of $P\left(K_{m}^{+}, S_{d}^{*} / S_{L}^{0}(\bar{Z})\right)$ 
in Fig. 12 and since the partial premixing acts to decrease $S_{d}^{*}$, the negative correlation is strengthened by excluding the partial premixing contributions. The value of correlation coefficient decreases to -0.43 from -0.37 when the partial premixing terms are excluded. At other positions with small contributions from the partial premixing, the joint pdfs, $P\left(K_{m}^{+}, S_{d}^{*} / S_{L}^{0}(\widetilde{Z})\right)$ and $P\left(K_{m}^{+}, S_{d}^{0} / S_{L}^{0}(\widetilde{Z})\right)$, remain similar with almost no change to the correlation coefficient noted earlier. This was also noted by Malkeson and Chakraborty (2010) for stratified flames.

To summarise, the effects of partially premixing on the displacement speed is nonnegligible at downstream positions and they act to reduce the negative correlation between the displacement speed and curvature. Also, these effects do not influence the alignment characteristics of the progress variable gradient with the principal strain directions observed for turbulent premixed flames. Next, we like to consider the question on negative flame stretch, ie., can $\langle\Phi\rangle_{s}$ in Eq. (1) be negative in mixed mode combustion or not? If so, what are the causes?

\subsection{Flame Surface Density and flame stretch}

As noted in Eq. (2), the behaviour of the flame stretch $\Phi$ depends on both $a_{T}$ and $2 S_{d} K_{m}$. The pdf of flame stretch computed using Eq. (2) is shown in Fig. 14 for few radial and axial locations. The flame stretch is normalised using the flame time of stoichiometric premixed unstrained planar flame, ie. $\Phi^{+}=\Phi \delta_{L, s t}^{0} / S_{L, s t}^{0}$. The locations chosen are within the flame brush and they are for $T_{32}>0, R / D=2$ and 2.5 for axial locations B-B and C-C respectively, and $T_{32}<0 R / D=3$ and 4 respectively for the B-B and C-C locations (see Fig. 6). The pdf peak is near zero which does not imply that the mean is zero. The pdf shows a long positive tail for locations with $T_{32}>0$ whereas for locations with $T_{32}<0$ the pdf has a long negative tail. Just to remind ourselves, $T_{32}>0$ implies the generation of FSD and $T_{32}<0$ implies the destruction of FSD by turbulence straining.

In general, the instantaneous flame stretch is both positive and negative as has been noted in many earlier studies (Rutland and Trouvé, 1993; Bray and Cant, 1991; Chen and Im, 1998; Nye et al., 1996; Renou et al., 1998; Kostiuk and Bray, 1993). However, these studies suggested that there is $50 \%$ or less probability for the flame stretch to be negative and this probability can be calculated by integrating the pdf shown in Fig. 14 from $-\infty$ to 0 . These integrated values are 0.44 and 0.57 respectively for $R / D=2$ and 3 at the axial position $\mathrm{B}-\mathrm{B}$. This integral takes a value of 0.41 and 0.6 respectively for $R / D=2.5$ and 4 at the axial position $\mathrm{C}-\mathrm{C}$. These integral values for locations with $T_{32}>0$ are in general agreement with observations made in earlier studies. However, this probability larger than $50 \%$ is observed for locations with $T_{32}<$ 0 , where the progress variable gradient predominantly aligns with the most extensive strain. These large probabilities for negative flame stretch are bound to yield negative mean value. Figure 15 depicting the radial variation of normalised surface averaged flame stretch, $\langle\Phi\rangle_{s}^{+}$, indeed shows this. The positive and negative values for $\langle\Phi\rangle^{+}$and the general trend were found to be retained when the sample size was reduced by 
half. A comparison of this figure to Fig. 6 suggests that $\langle\Phi\rangle_{s}^{+}$is negative in regions with $T_{32}<0$ for the lifted flame considered in this study. The mean flame stretch is $\langle\Phi\rangle^{+}=\left\langle a_{T}\right\rangle^{+}+2\left\langle S_{d}^{*} K_{m}\right\rangle^{+}$and the discussion in the previous subsection showed that $\left\langle S_{d}^{*} K_{m}\right\rangle^{+}<0$. Hence, to have $\langle\Phi\rangle^{+}>0$ one must have positive $\left\langle a_{T}\right\rangle^{+}$and $\left|\left\langle a_{T}\right\rangle^{+}\right|>$ $\left|\left\langle S_{d}^{*} K_{m}\right\rangle^{+}\right|$. This is the case if $T_{32}>0$ yielding positive $\langle\Phi\rangle^{+}$. Since $\left\langle S_{d}^{*} K_{m}\right\rangle^{+}$is negative, the mean flame stretch can become negative only if $\left\langle a_{T}\right\rangle^{+}<0$ or $\left|\left\langle a_{T}\right\rangle^{+}\right|<\left|\left\langle S_{d}^{*} K_{m}\right\rangle^{+}\right|$. The second condition is obvious and the former is of interest here. Using Eq. (2) and the analysis of Eq. (10), one writes $\left\langle a_{T}\right\rangle^{+}=\nabla^{+} \cdot \mathbf{u}^{+}+\left\langle T_{32}^{+}\right\rangle$. Thus, one must have $\left\langle T_{32}^{+}\right\rangle<0$ and $\left|\left\langle T_{32}^{+}\right\rangle\right|>\nabla^{+} \cdot \mathbf{u}^{+}$to have negative $\left\langle a_{T}{ }^{+}\right\rangle$. Physically, this implies that the turbulence-scalar interaction effect is stronger than the dilatation effect on the flame stretch, suggesting that the flame stretch due to turbulent straining can be negative if the scalar gradient vector aligns with the most extensive principal strain as has been observed in this study (see Figs. 8, 14 and 15). Further evidences are provided later while discussing Fig. 17

To elucidate the above observation, one writes Eq. (1) as

$$
\frac{1}{\Sigma} \frac{\mathrm{d} \Sigma}{\mathrm{d} t}=\langle\Phi\rangle_{s}-\frac{\partial\left\langle u_{j}+S_{d} n_{j}\right\rangle_{s}}{\partial x_{j}}
$$

after some simple rearrangement. It is worth noting that the second term in the right hand side of Eq. (15) is $(\mathrm{d}(\delta V) / \mathrm{d} t) / \delta V$ as shown by Candel and Poinsot (1990) where $\delta V$ is the elemental fluid volume following the flame element and thus, this term is positive. The role of the alignment characteristics can be seen by writing the above equation, after using Eq. (2), as

$$
\begin{aligned}
\frac{1}{\Sigma} \frac{\mathrm{d} \Sigma}{\mathrm{d} t} & =-\left\langle n_{i} n_{j} \frac{\partial u_{i}}{\partial x_{j}}\right\rangle_{s}-\left\langle n_{i} \frac{\partial S_{d}}{\partial x_{i}}\right\rangle_{s} \\
& =\underbrace{-\left\langle\alpha \cos ^{2} \theta_{\alpha}+\beta \cos ^{2} \theta_{\beta}+\gamma \cos ^{2} \theta_{\gamma}\right\rangle_{s}}_{\widehat{T_{1}}} \underbrace{-\left\langle n_{i} \frac{\partial S_{d}}{\partial x_{i}}\right\rangle_{s}}_{\widehat{T_{2}}} .
\end{aligned}
$$

It is to be noted that $S_{d}=S_{d}^{*}$ for the above equations. The following observations are made using the second line of Eq. (16):

1. For a material surface $S_{d}=0$ and the alignment is known to be with $\gamma$ strain and thus $\mathrm{d} \Sigma / \mathrm{d} t=\langle|\gamma|\rangle_{s} \Sigma$ resulting in an exponential growth of the surface density. This is well known from numerous studies in the past.

2. For a propagating passive surface with a constant displacement speed, one observes the exponential growth of $\Sigma$ with $t$ as for the material surface. This growth due to turbulent straining has also been noted while analysing $G$ equation closure (Peters, 1999) and turbulent flame speed analysis (Kerstein, 1988). It is known that constant $S_{d}^{*}$ leads to the possibility of cusp formation resulting from possible self intersection of the surface (Pope, 1988, Kollmann and Chen, 1994, 1998). 
The surface density transport equation is known to be singular when the surface interactions occur (Pope, 1988; Kollmann and Chen, 1998) and the above analysis does not hold. When the molecular diffusion is present, which is so in real situations, the displacement speed is less likely to be constant (see Eq. 4) and the possibility for the cusp formation is reduced and $\Sigma$ will not grow exponentially.

3. For high Damkohler number premixed flames with unity Lewis number one gets $\mathrm{d} \Sigma / \mathrm{d} t=-\langle\alpha\rangle_{s} \Sigma$ since the scalar gradient alignment is expected to be with the most extensive strain. This leads to the loss of FSD. This effect is not included in the FSD models proposed in past studies. The turbulence effects dominate in regions with small heat release and thus one observes the growth of $\Sigma$ for these regions. These effects are compounded by the response of the displacement speed to the curvature in non-unity Lewis number flames (Bradley, 2002).

4. The balance of contributions from $\widehat{T}_{1}$ and $\widehat{T}_{2}$ determines the temporal variation of $\Sigma$ as per Eq. (16) in mixed mode combustion. If one presumes a flamelet type combustion in these flames then $\widehat{T_{2}}$ can be shown to be $\widehat{T_{2}}=-\left\langle\left(\mathrm{d} S_{L} / \mathrm{d} Z\right)(\nabla c \cdot \nabla Z) /|\nabla c|\right\rangle_{s}$. Now, one realises the role of the cross dissipation on the surface averaged flame stretch in the mixed mode combustion.

The contributions of $\widehat{T}_{1}$ and $\widehat{T_{2}}$ can be obtained from the DNS data, however one must recognise that it is not straight forward to calculate $\mathrm{d} \Sigma / \mathrm{d} t$ following a flame element. Before discussing the results on these two terms, let us first study temporal variation of $\Sigma$ at locations B1 and $\mathbf{C 1}$, which are inside the flame brush as in Fig. 3. This and other statistics presented in this section for these two locations are constructed following the second method of data processing described in section 2 .

The FSD in Eq. (1) can be calculated directly from the DNS data using (Pope, 1988, 1990; Vervisch et al., 1995)

$$
\Sigma\left(c^{*}\right)=\left\langle\mid \nabla c \| c=c^{*}\right\rangle \bar{P}\left(c^{*}\right),
$$

where $\bar{P}$ is the marginal pdf of $c$. The variations of $c$ at the two locations $\mathbf{B} 1$ and $\mathbf{C 1}$ over the sampling period yield the Favre RMS values less than 5\%. Strictly, one must do conditional averaging as per Eq. (17). Since the variation of $c$ at these two locations are small and the sample size is insufficient to construct the conditional mean, the FSD values are calculated by averaging over the sampling period and these values are taken to be for single $c^{*}$ value, which is about 0.89 for $\mathbf{B 1}$ and 0.93 for $\mathbf{C 1}$.

The temporal evolution of $\Sigma^{+}=\Sigma \delta_{L}^{0}(\widetilde{Z})$ is shown in Fig. 16 a for the two locations, B1 and C1, inside the flame brush. Just to recall, these two particular positions are chosen because of the preferential alignment of the scalar gradient with the most extensive principal strain rate as noted in Fig. 8 . The time in Fig. 16 is normalised using the eddy turnover time at $\mathbf{B} 1$, calculated as $k^{3 / 2} \sqrt{\varepsilon}$ with the Favre averaged turbulent kinetic energy, $\widetilde{k}$, and its dissipation rate, $\widetilde{\varepsilon}$, obtained over the period marked with a small rectangle in Fig. 16a. The horizontal axis on the top side of this figure indicates 
the time normalised using the eddy turnover time at the location $\mathbf{C 1}$. The initial sharp drop of $\Sigma^{+}$for the location $\mathbf{B} 1$ and its sharp rise at about $\widehat{t}=1$ for the location $\mathbf{C 1}$ might be due to initial numerical transient in the simulation since the 200 million grid simulation used in this study was started using the results of a simulation with 23 million grid points used by Mizobuchi et al. (2002, 2005) In order to minimise the effects of initial transients, the period marked with the small rectangle is chosen for the analysis of negative flame stretch.

A close-up view of the temporal variation of $\Sigma^{+}$for the chosen period of analysis is shown in Figs. 16p for the location B1 and 16 for the location C1. The temporal derivative, $\mathcal{T}^{+}=\left(\partial \Sigma^{+} / \partial t^{+}\right) / \Sigma^{+}$, is also shown. This quantity takes both positive and negative values and its negative value is of interest here. As noted earlier, computing the Lagrangian derivative is not easy and thus the Eulerian frame is chosen for further analysis. By simply rearranging Eq. (1) one writes $\langle\Phi\rangle_{s}=\mathcal{T}+\left[\nabla \cdot\left\langle\left(\mathbf{u}+S_{d} \mathbf{n}\right)\right\rangle_{s} \Sigma\right] / \Sigma$. In this expression, the convective term is a transport term since it appears as a divergence and thus the behaviour of $\mathcal{T}^{+}$has direct bearing on the behaviour of the flame stretch $\langle\Phi\rangle_{s}^{+}$.

As noted earlier in this section and in Eq. (2) the flame stretch has two contributions and their variations are shown in Figs. $17 \mathrm{a}$ and $17 \mathrm{p}$ respectively for the locations $\mathbf{B} 1$ and $\mathbf{C 1}$. These quantities are normalised as indicated in the figure caption. The tangential strain rate is expected to be positive in general but negative values are observed for the location C1. To ascertain the physics discussed earlier in this section for this negative $\left\langle a_{T}\right\rangle_{s}^{+}$, the surface averaged value of the normal strain rate, $\widehat{T_{1}^{+}}$in Eq. $(16$, , is also shown in Fig.17. It is clear that the magnitude of $\nabla^{+} \cdot \mathbf{u}^{+}$contribution to the tangential strain is small and the behaviour of $\left\langle a_{T}\right\rangle_{s}^{+}$is dominated by $\widehat{T_{1}^{+}}$behaviour. One must note that the scalar gradient alignment characteristics dictates the behaviour of $\widehat{T_{1}^{+}}$. A gradual change of the scalar gradient alignment with the most extensive strain to the compressive strain was observed in the analysis (not shown), which causes $\left\langle a_{T}\right\rangle_{s}^{+}$to gradually change from negative to positive value as shown in Fig. $17 \mathrm{~b}$ for the location C1. There is no such interesting changes at the location B1 since $\left\langle a_{T}\right\rangle_{s}^{+}>0$ over the period of analysis.

The contribution of the curvature related term shown in Fig. 17 is predominantly negative as one would expect from the joint pdf results discussed earlier in section 3.4 . Also, the negative values are much larger than the positive values of $\left\langle a_{T}\right\rangle_{s}^{+}$resulting in the negative flame stretch. The variation of the cross dissipation related term, $\widehat{T_{2}^{+}}$ in Eq. (16), shown in Fig. 17 suggests that its contribution is small for the conditions of the flame analysed here. The variation of equivalence ratio at the location $\mathbf{B 1}$ is very small but for the location $\mathbf{C 1}$ it varies in the range $0.83 \leq \phi \leq 1.52$. Despite this large variation, the small contribution of $\widehat{T_{2}^{+}}$for this location is because of small cross dissipation rate (non-alignment of $\nabla c$ and $\nabla Z$ ).

It is expected that the balance of various source and sink terms similar to that shown here to hold appropriately if one moves the observation window to another instant (see Fig. 16a). From the results shown in Fig. 17, it is apparent that the bulk of the 
negative values of the surface averaged flame stretch results from the curvature related term. However, there are instances with the curvature related term is close to zero and negative values for the turbulent straining. Earlier studies on turbulent premixed flames showed that the mean of turbulent straining is positive and results in positive flame stretch. Here, the negative stretch due to turbulent straining is observed in the mixed mode combustion. The partial premixing is observed to reduce the negative correlation between the displacement and curvature in subsection 3.4 and thus one can infer that the contribution of turbulent straining to the negative flame stretch is enhanced in the mixed mode combustion.

\section{Conclusion}

DNS data of a turbulent lifted hydrogen jet flame, simulated by Mizobuchi et al. (2002, 2005), has been analysed to shed physical insight on the behaviour of flame stretch dynamics and its relation to other physical processes, in particular, the turbulence-scalar interaction and partial premixing effects. This lifted flame is a good example for mixed mode combustion since the fuel is mixed with quiescent air by the jet entrainment and turbulence processes in the near field of the fuel jet. This mixing process is unsteady creating spatio-temporally inhomogeneous mixture resulting in mixed mode combustion. A combined analysis of instantaneous fuel consumption rate and flame index suggests the coexistence of premixed and non-premixed combustion modes as has been observed by Mizobuchi et al. (2002, 2005). However, the averaged fuel consumption rate and flame index demonstrates that the premixed combustion with variable equivalence ratio is predominant and non-premixed combustion occurs only in a narrow region at the edge of the jet shear layer as shown in Fig. 4.

The mixed mode combustion can be modelled by combining contributions from non-premixed and premixed flamelets appropriately as has been done by Domingo et al. (2002). The FSD based approach is popular approach for premixed combustion and this approach need closure models for the flame stretch. The flame stretch includes contributions from the displacement speed-curvature correlation and tangential strain rate induced by turbulence. These quantities are analysed using the DNS data in this study. The displacement speed-curvature correlation is observed to be negative in this mixed mode combustion, which is consistent with previous studies. The effects of partial premixing on this correlation is observed to be non-negligible and they act to reduce this negative correlation. Both positive and negative mean flame stretch have been observed in this study. The usual positive mean flame stretch results from strong positive contribution from the turbulent straining $\left(\left\langle a_{T}\right\rangle\right.$, see Eq. 2) and the negative values for the mean flame stretch is observed when the turbulent straining contribution is negative. This negative contribution is shown to result from the alignment of the scalar gradient (flame normal) with the most extensive principal strain rate, which dictates the turbulence-scalar interaction dynamics. This negative contribution to the mean flame stretch has also been elucidated through a simple analysis of the FSD 
transport equation. The partial premixing seem to enhance the contribution of turbulent straining to the negative flame stretch by reducing the negative correlation between the displacement speed and curvature. These observations have implications for SGS combustion model development and the currently available models do not cater for the negative values of surface averaged flame stretch observed in this study. The modelling avenues to include these effects in SGS combustion model will be explored in future studies.

\section{Acknowledgments}

The authors are grateful for the inspiring discussion with Prof. K.N.C. Bray, and financial support from Mitsubishi Heavy Industries (MHI) is gratefully acknowledged. A part of this work is performed under the collaborative research between Cambridge University and JAXA. 


\section{References}

Anselmo-Filho, P., Hochgreb, S., Barlow, R. S., and Cant, R. S. 2009. Experimental measurements of geometric properties of turbulent stratified flames. Proc. Combust. Inst., 32, 1763-1770.

Bilger, R. W. 1988. The structure of turbulent nonpremixed flames. Proc. Combust. Inst., 22, 475-488.

Bradley, D. 2002. Problems of predicting turbulent burning rates. Combust. Theory Model., 6, 361-382.

Bray, K. N. C., and Cant, R. S. 1991. Some applications of Kolmogorov's turbulence research in the field of combustion. Proc. R. Soc. Lond. A, 434, 217-240.

Bray, K. N. C., Domingo, P., and Vervisch, L. 2005. Role of the progress variable in models for partially premixed turbulent combustion. Combust. Flame, 141, 431437.

Candel, S. M., and Poinsot, T. J. 1990. Flame stretch and the balance equation for flame surface area. Combust. Sci.Tech., 70 (1-3), 1-15.

Cant, R. S. 2011. RANS and LES modelling of premixed turbulent combustion. In Turbulent Combustion Modeling ((Eds.) T. Echekki, and E. Mastorakos). SpringerVerlag, Heidelberg.

Chakarborty, N., and Cant, R. S. 2005. Effects of strain rate and curvature on surface density function transport in turbulent premixed flames in the thin reaction zones regime. Phys. Fluids, 17, 065108.

Chakraborty, N., Klein, M., and Swaminathan, N. 2009. Effects of Lewis number on the reactive scalar gradient alignment with local strain rate in turbulent premixed flames. Proc. Combust. Inst., 32, 1409-1417.

Chakraborty, N., Rogerson, J. W., and Swaminathan, N. 2010. The scalar gradient alignment statistics of flame kernels and its modelling implications for turbulent premixed combustion. Flow Turbulence Combust., 85, 25-55.

Chakraborty, N., and Swaminathan, N. 2007a. Influence of the Damköhler number on turbulence-scalar interaction in premixed flames. I. physical insight. Phys. Fluids, 19, 045103.

Chakraborty, N., and Swaminathan, N. 2007b. Influence of the Damköhler number on turbulence-scalar interaction in premixed flames. II. model development. Phys. Fluids, 19, 045104. 
Chen, J. H., and Im, H. G. 1998. Correlation of flame speed with stretch in turbulent premixed methane/air flames. Proc. Combust. Inst., 27, 819-826.

Chen, J. H., and Im, H. G. 2000. Stretch effects on the burning velocity of turbulent premixed hydrogen/air flames. Proc. Combust. Inst., 28, 211-218.

Cheng, T.S., Wehrmeyer, J.A., and Pitz, R.W. 2007. Conditional analysis of lifted hydrogen jet diffusion flame experimental data and comparison to laminar flame solutions. Combust. Flame, 150, 340-354.

Cheng, T.S., Wehrmeyer, J. A., and Pitz, R. W. 1992. Simultaneous temperature and multispecies measurement in a lifted hydrogen diffusion flame. Combust. Flame, 91, 323-345.

Dold, J. W. 1989. Flame propagation in a nonuniform mixture: Analysis of a slowly varying triple flame. Combust. Flame, 76, 71-88.

Domingo, P., Vervisch, L., and Bray, K. N. C. 2002. Partially premixed flamelets in LES of non-premixed turbulent combustion. Combust. Theory Model., 6, 529-551.

Echekki, T., and Chen, J. H. 1996. Unsteady strain rate and curvature effects in turbulent premixed methane-air flames. Combust. Flame, 106, 184-202.

Gonzalez, M., and Paranthoën, P. 2011. Effect of variable mass density on the kinematics of scalar gradient. Phy. Fluids, 23, 075107.

Gran, I. R., Echekki, T., and Chen, J. H. 1996. Negative flame speed in an unsteady 2-D premixed flame: A computational study. Proc. Combust. Inst., 26, 323-329.

Hartung, G., Hult, J., Kaminski, .C. F., Rogerson, J. W., and Swaminathan, N. 2008. Effect of heat release on turbulence and scalar-turbulence interaction in premixed combustion. Phys. Fluids, 20, 035110.

Hawkes, E. R., and Chen, J. H. 2005. Evaluation of models for flame stretch due to curvature in the thin reaction zones regime. Proc. Combust. Inst., 30, 647-655.

Helié, J., and Trouvé, A. 1998. Turbulent flame propagation in partially premixed combustion. Proc. Combust. Inst., 27, 891-898.

Kerstein, A. R. 1988. Simple derivation of Yakhot's turbulent premixed flamespeed formula. Combust. Sci. Technol., 60, 163-165.

Kim, S. H., and Pitsch, H. 2007. Scalar gradient and small-scale structure in turbulent premixed combustion. Phy. Fluids, 19, 115104, 1-14.

Kioni, P. N., Rogg, B., Bray, K. N. C., and Linán, A. 1993. Flame spread in laminar mixing layers: The triple flame. Combust. Flame, 95, 277-290. 
Kollmann, W., and Chen, J. H. 1994. Dynamics of the flame surface area in turbulent non-premixed combustion. Proc. Combust. Inst., 25, 1091-1098.

Kollmann, W., and Chen, J. H. 1998. Pocket formation and the flame surface density equation. Proc. Combust. Inst., 27, 927-934.

Kostiuk, L. W., and Bray, K. N. C. 1993. Mean effects of stretch on laminar flamelets in a premixed turbulent flame. Combust. Sci. Tech., 95, 193-212.

Law, C. K. 2006. Combustion Physics. Cambridge University Press, Cambridge, UK, Cambridge University Press, Cambridge, UK.

Luo, Z., Yoo, C. S., Richardson, E. S., Chen, J. H., Law, C. K., and Lu, T. 2012. Chemical explosive mode analysis for a turbulent lifted ethylene jet flame in highlyheated coflow. Combust. Flame, 159, 265-274.

Malkeson, Sean P., and Chakraborty, Nilanjan. 2010. Statistical analysis of displacement speed in turbulent stratified flames: A direct numerical simulation study. Combust. Sci.Tech., 182 (11-12), 1841-1883.

Malkeson, Sean P., and Chakraborty, Nilanjan. 2011. Alignment statistics of active and passive scalar gradients in turbulent stratified flames. Phys. Rev. E, 83, 046308.

Mizobuchi, Y., Shinjo, J., Ogawa, S., and Takeno, T. 2005. A numerical study on the formation of diffusion flame islands in a turbulent hydrogen jet lifted flame. Proc. Combust. Inst., 30, 611-619.

Mizobuchi, Y., Tachibana, S., Shinio, J., Ogawa, S., and Takeno, T. 2002. A numerical analysis of the structure of a turbulent hydrogen jet lifted flame. Proc. Combust. Inst., 29, 2009-2015.

Mura, A., Robin, V., Champion, M., and Hasegawa, T. 2009. Small scale features of velocity and scalar fields in turbulent premixed flames. Flow Turbulence Combust., 82, 339-358.

Mura, A., Tsuboi, K., and Hasegawa, T. 2008. Modelling of the correlation between velocity and reactive scalar gradients in turbulent premixed flames based on DNS data. Combust. Theory. Model., 12, 671.

Nye, D. A., Lee, J. G., Lee, T.-W, and Santavicca, D. A. 1996. Flame stretch measurements during the interaction of premixed flames and Kármán vortex streets using PIV. Combust. Flame, 105, 167-179.

Peters, N. 1999. The turbulent burning velocity for large-scale and small-scale turbulence. J. Fluid Mech., 384, 107-132. 
Plessing, T., Terhoeven, P., Peters, N., and Mansour, M. S. 1998. An experimental and numerical study of a laminar triple flame. Combust. Flame, 115, 335-353.

Pope, S. B. 1988. The evolution of surfaces in turbulence. Int. J. Engng. Sci., 5, 445469.

Pope, S. B. 1990. Computations of turbulent combustion: Progress and challenges. Proc. Combust. Inst., 23, 591-612.

Renou, B., Boukhalfa, A., Puechberty, D., and Trinité, M. 1998. Effects of stretch on the local structure of freely propagating premixed low-turbulent flames with various Lewis numbers. Proc. Combust. Inst., 27, 841-847.

Richardson, E. S., Sankaran, R., Grout, R. W., and Chen, J. H. 2010. Numerical analysis of reaction-diffusion effects on species mixing rates in turbulent premixed methane-air combustion. Combust. Flame, 157, 506-515.

Ruan, S., Swaminathan, N., Bray, K. N. C., Mizobuchi, Y., and Takeno, T. 2012. Scalar and its dissipation in the near field of turbulent lifted jet flame. Combust. Flame, 159, 591-608.

Ruetsch, G. R., Vervisch, L., and Linán, A. 1995. Effects of heat release on triple flames. Phys. Fluids, 7 (6), 1447-1454.

Rutland, C. J., and Trouvé, A. 1993. Direct simulations of premixed turbulent flames with nonunity Lewis numbers. Combust. Flame, 94, 41-57.

Smooke, M. D., and Givangoli, V. 1991. Formulation of the premixed and nonpremixed test problems. In Reduced Kinetic Mechanisms and Asymptotic Approximations for Methane-air Flames ((Ed.) M. D. Smooke), Lecture Notes in Physics, vol. 384. Springer-Verlag, Berlin.

Steinberg, A. M., Driscoll, J. F., and Swaminathan, N. 2012. Statistics and dynamics of turbulence-flame alignment in premixed combustion. Combust. Flame, 159, 25762588 .

Swaminathan, N., and Grout, R. W. 2006. Interaction of turbulence and scalar fields in premixed flames. Phys. Fluids, 18 (4), 045102.

Trouvé, A., and Poinsot, T.J. 1994. The evolution equation for the flame surface density in turbulent premixed combustion. J. Fluid Mech., 278, 1-31.

Vervisch, L., Bidaux, E., Bray, K. N. C., and Kollmann, W. 1995. Surface density function in premixed turbulent combustion modeling, similarities between probability density function and flame surface approaches. Phys.Fluids, 7 (10), 2496-2503. 
Veynante, D., and Vervisch, L. 2002. Turbulent combustion modelling. Prog. Energy Combust. Sci., 28, 193-266.

Westbrook, C. K. 1982. Hydrogen oxidation kinetics in gaseous detonations. Combust. Sci. Technol., 29, 67-82.

Yamashita, H., Shimada, M., and Takeno, T. 1996. A numerical study on flame stability at the transition point of jet diffusion flames. Proc. Combust. Inst., 26, 27-34.

Yoo, C. S., Sankaran, R., and Chen, J. H. 2009. Three-dimensional direct numerical simulation of a turbulent lifted hydrogen jet flame in heated coflow: flame stabilization and structure. J. Fluid Mech., 640, 453-481. 


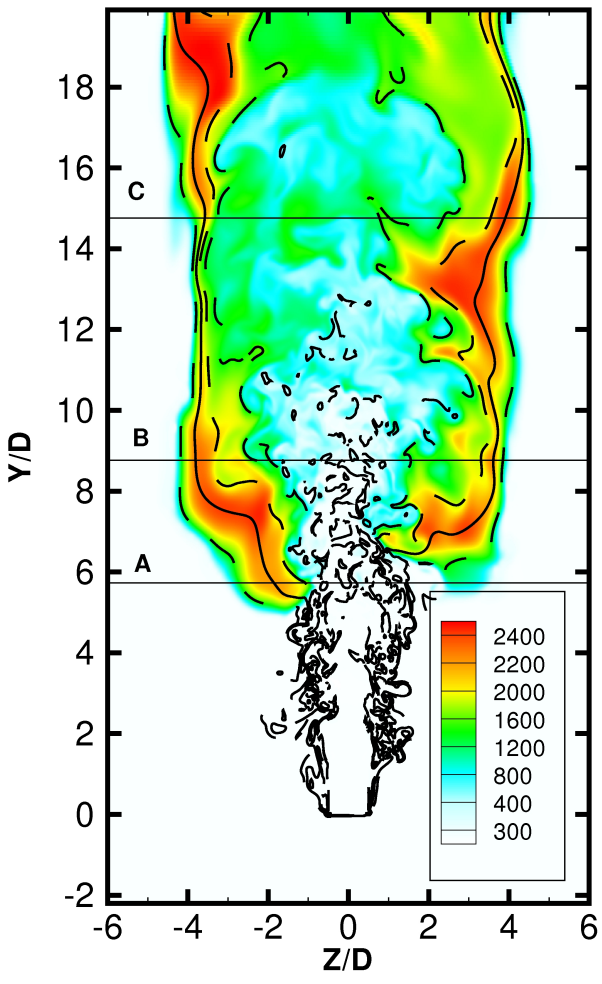

(b)

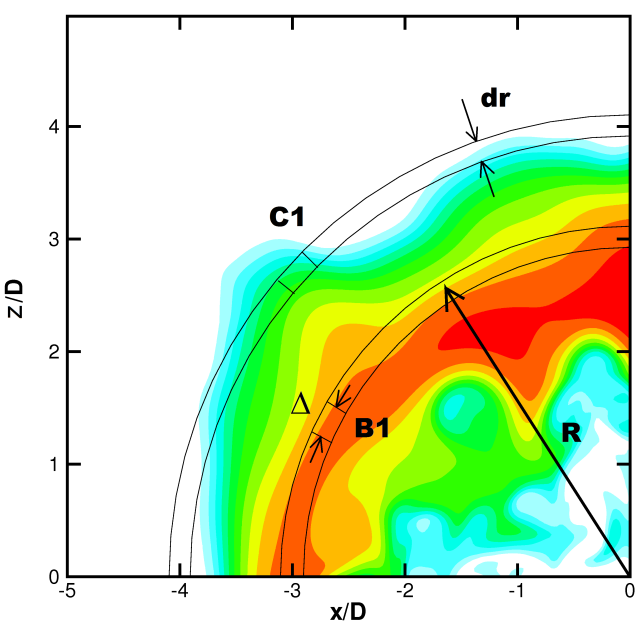

Figure 1: Turbulent jet flame configuration in the DNS. (a) Instantaneous temperature (K) field in mid $z-y$ plane. Solid black line is stoichiometric mixture fraction contour $Z_{s t}=0.03$. Dash lines are mixture fraction contours for $0.01,0.05,0.10,0.20$ and 0.30 . (b) Illustration of data processing method, instantaneous temperature field in an arbitrary $z-x$ plane is shown with positions B1 and $\mathbf{C 1}$ highlighted. 


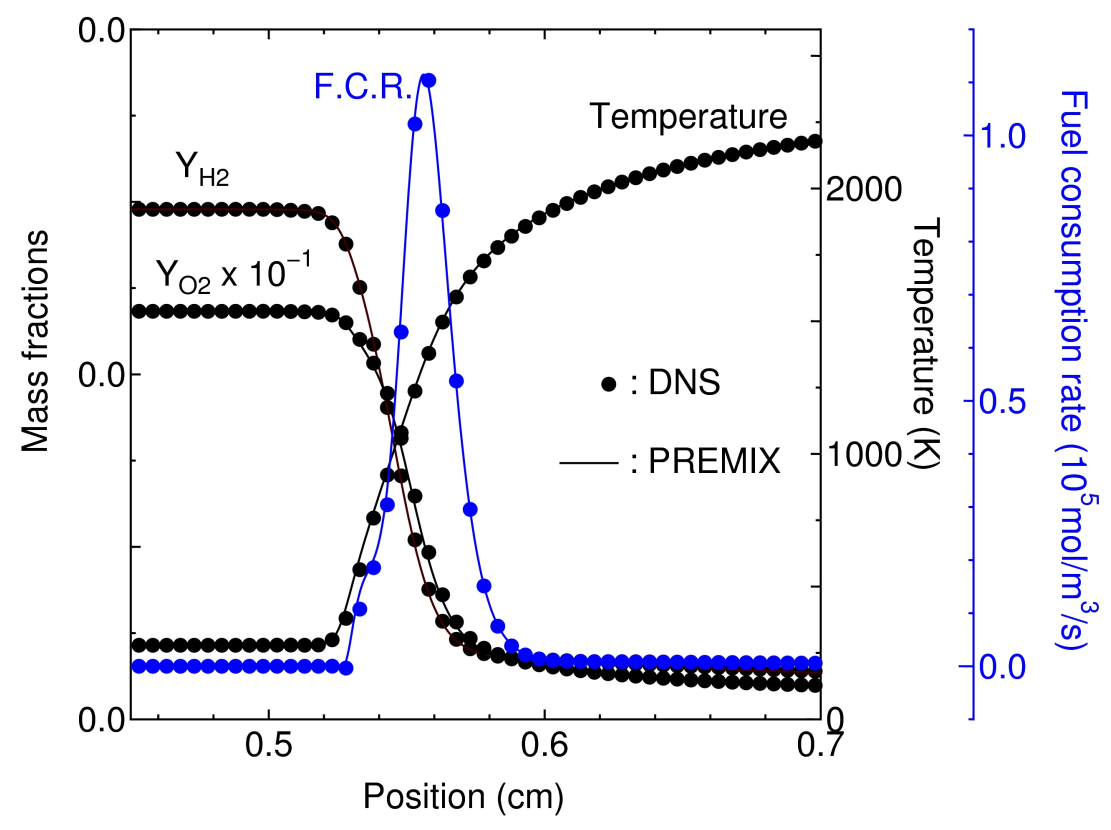

Figure 2: Comparison of stoichiometric H2/Air laminar flame computed using CHEMKIN and DNS codes. The spatial resolution of DNS is $0.05 \mathrm{~mm}$ and that of CHEMKIN-PREMIX is more than 20 times finer in the reacting region.
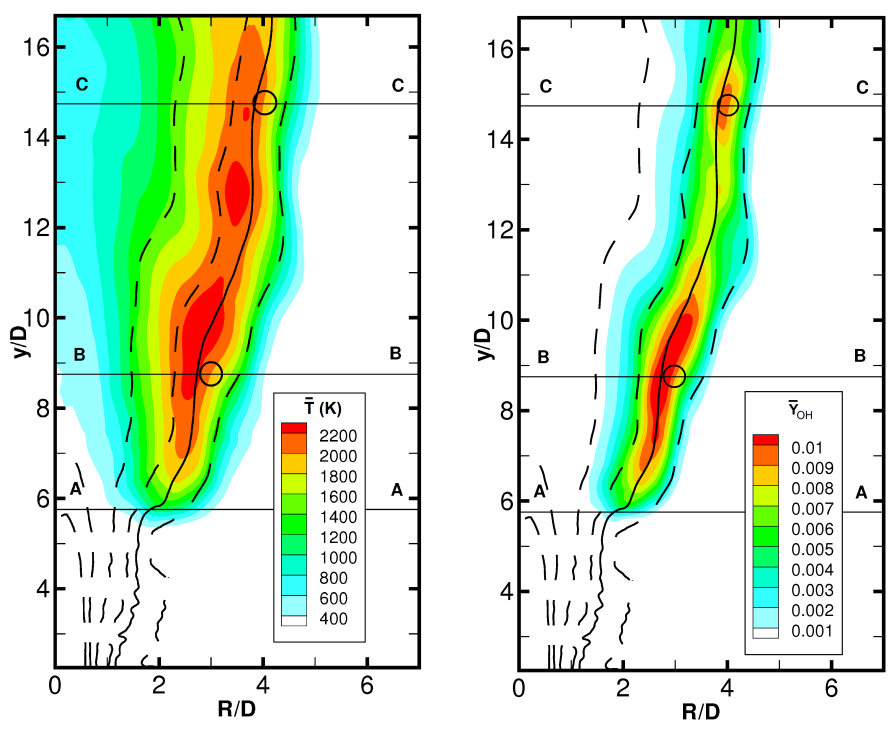

Figure 3: Spatial variation of Reynolds averaged $\mathrm{T}$ and $Y_{\mathrm{OH}}$. Solid black line is the stoichiometric mixture fraction contour $\bar{Z}=0.03$. Dash lines are the contours of $\bar{Z}=0.01,0.05,0.10,0.20$ and 0.30. Positions B1 and $\mathbf{C 1}$ are also highlighted. 

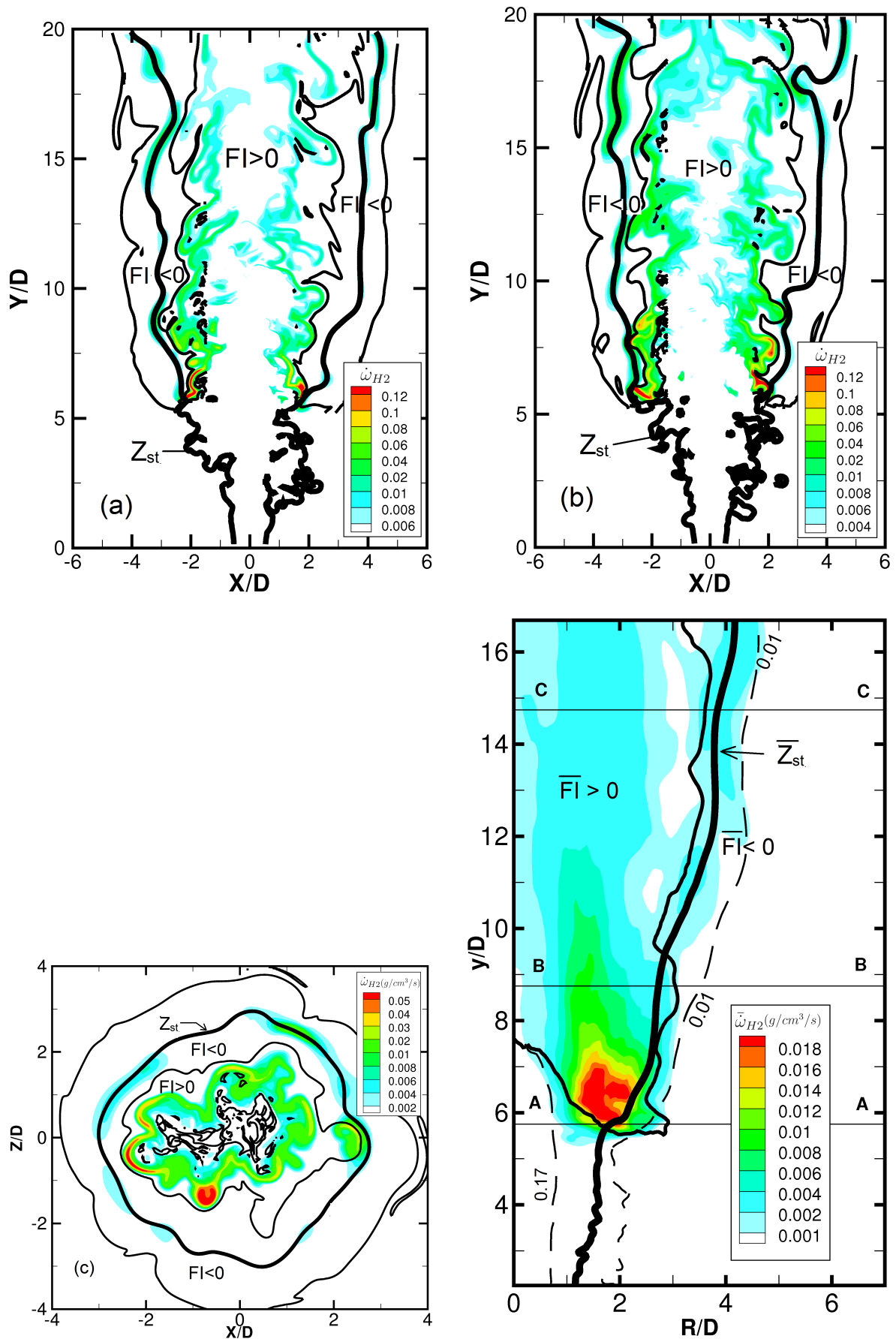

Figure 4: Typical variation of instantaneous fuel consumption rate $\left(\mathrm{g} / \mathrm{cm}^{3} / \mathrm{s}\right)$ in mid $x-y$ plane is shown in (a) and (b) for two arbitrarily chosen time. Figure (c) shows the variation in $x-z$ plane at $y=9 D$. The averaged fuel consumption rate $\left(\mathrm{g} / \mathrm{cm}^{3} / \mathrm{s}\right)$ is shown in (d). The thin lines are FI $=0$ contour. 

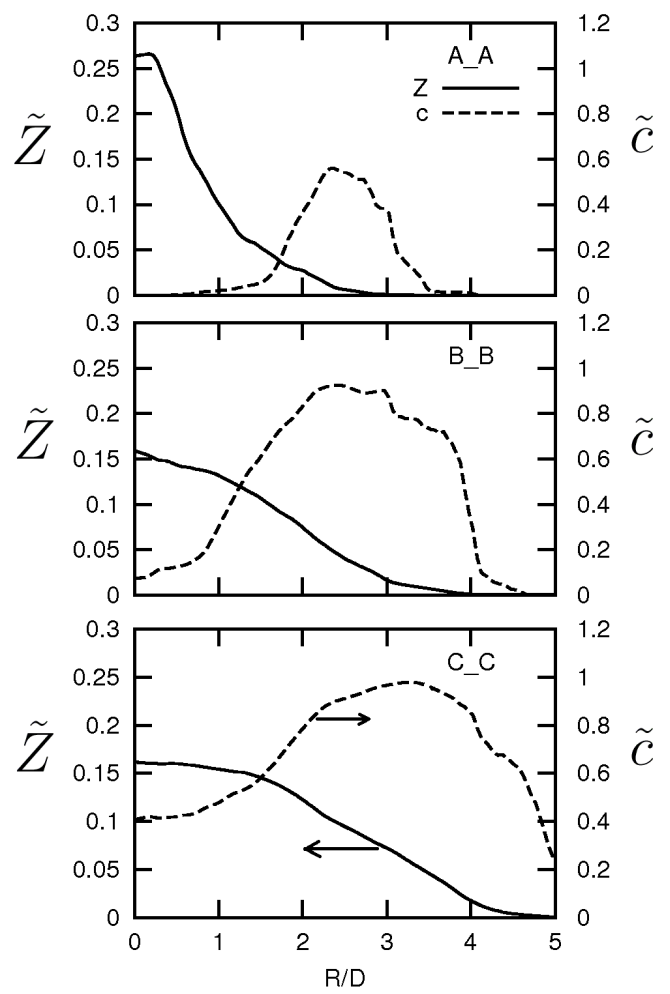

Figure 5: Radial variation of $\widetilde{Z}$ and $\widetilde{c}$ at 3 axial positions. 

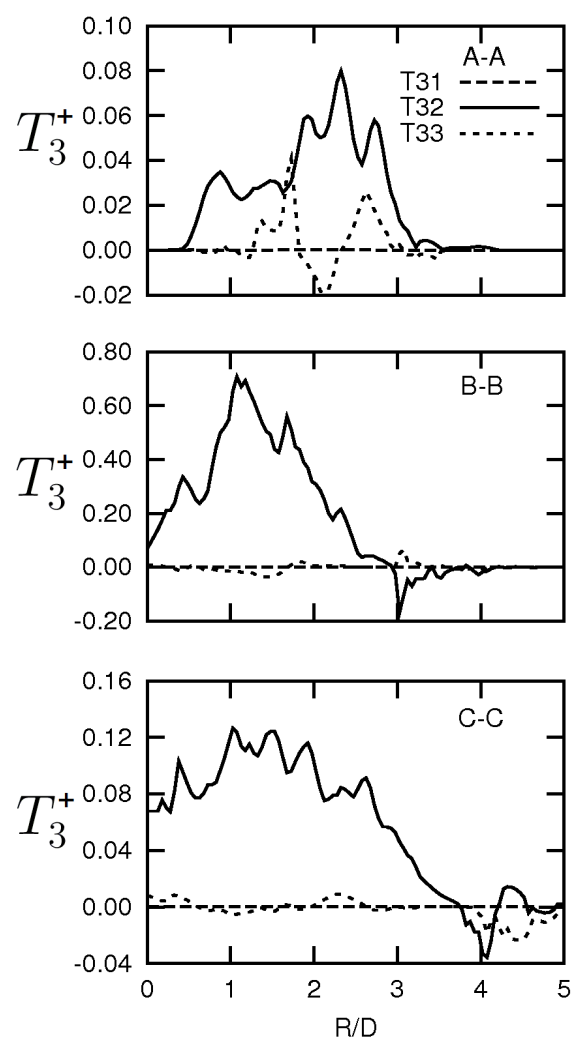

Figure 6: Typical radial variation of $T_{3}^{+}=T_{3} t_{f}^{2} / \rho_{u, s t}$, with $t_{f}=\delta_{L, s t}^{0} / S_{L, s t}^{0}$. Results are shown for three axial positions.

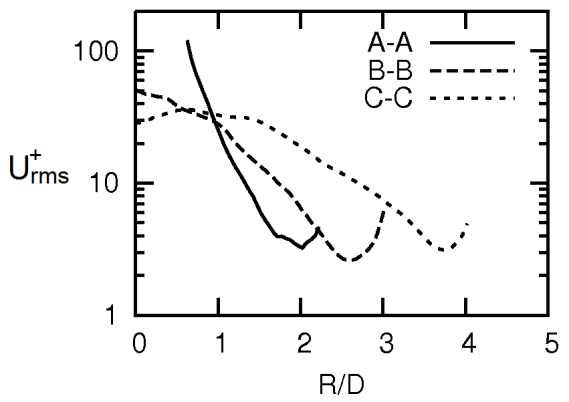

Figure 7: Typical radial variation of $U_{r m s}^{+}=U_{r m s} / S_{L}^{0}(\tilde{Z})$ for three axial locations. 

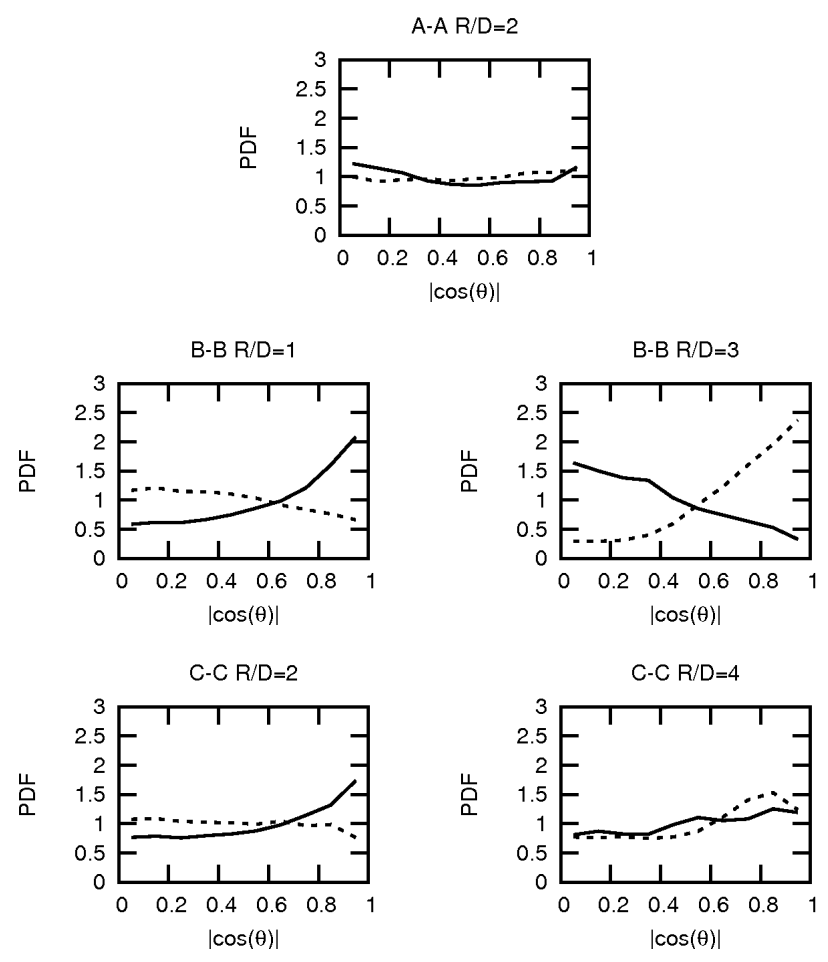

Figure 8: PDF of scalar gradient alignment with the principal compressive, $\gamma$ (solid) and extensive, $\alpha$ (dotted) strains at various axial and radial locations.

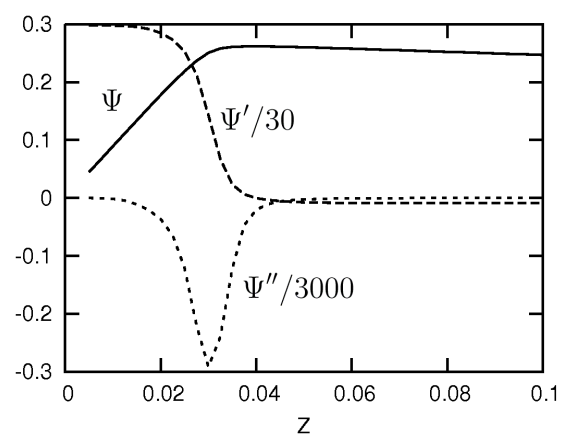

Figure 9: Variation of $\Psi=Y_{H 2 O}^{E q}, d \Psi / d Z$ and $d^{2} \Psi / d Z^{2}$ with the mixture fraction $Z$. 

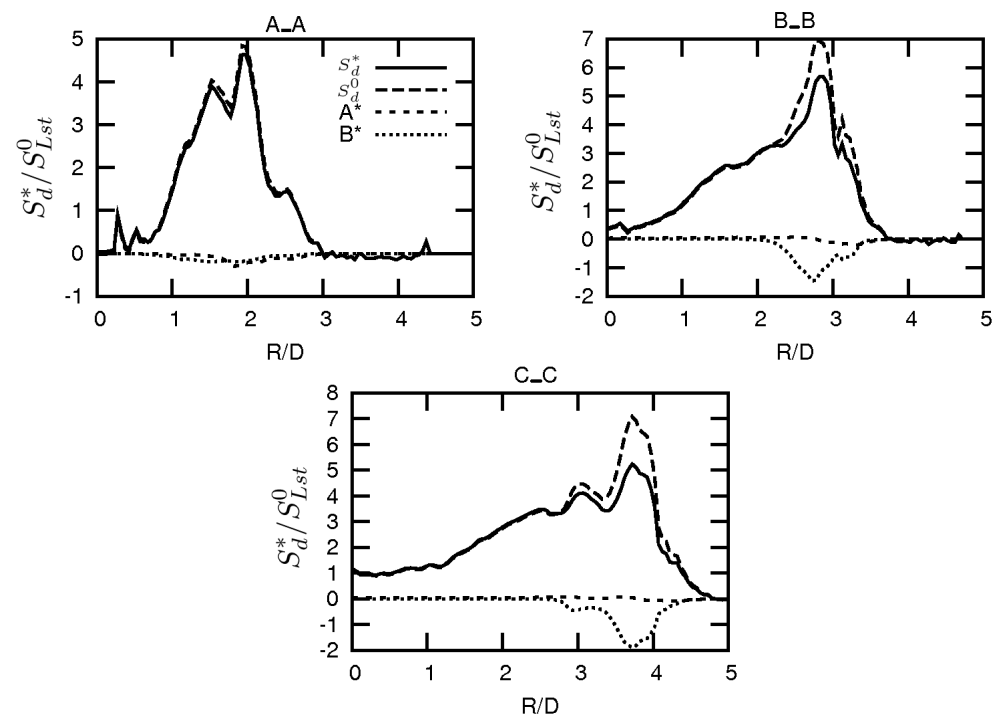

Figure 10: Radial variation of normalised displacement speed, $\left\langle S_{d}^{*}\right\rangle_{s} / S_{L, s t}^{0}$, and its components at three axial positions.

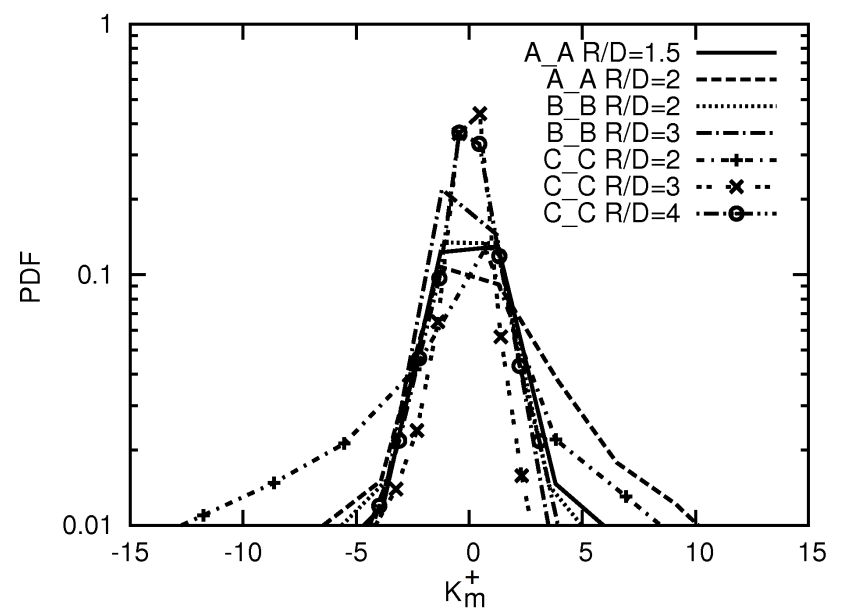

Figure 11: PDF of normalised curvature, $K_{m}^{+}=K_{m} \delta_{L}^{0}(\widetilde{Z})$, at various axial and radial locations. 

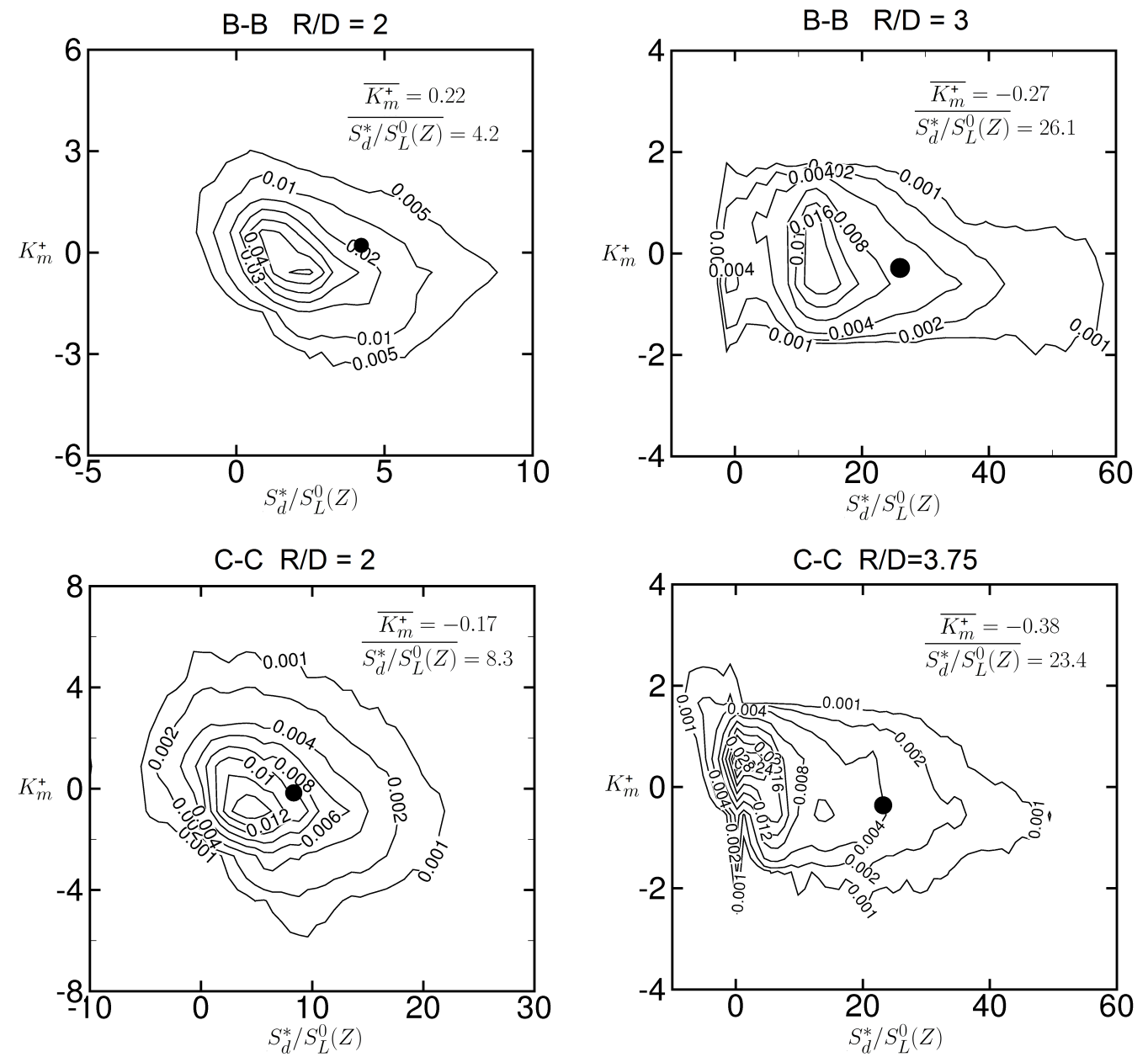

Figure 12: Joint PDFs of normalised curvature, $K_{m}^{+}$, and total displacement speed, $S_{d}^{*} / S_{L}^{0}(\widetilde{Z})$, for various axial and radial positions. Mean values are marked using solid circles. 


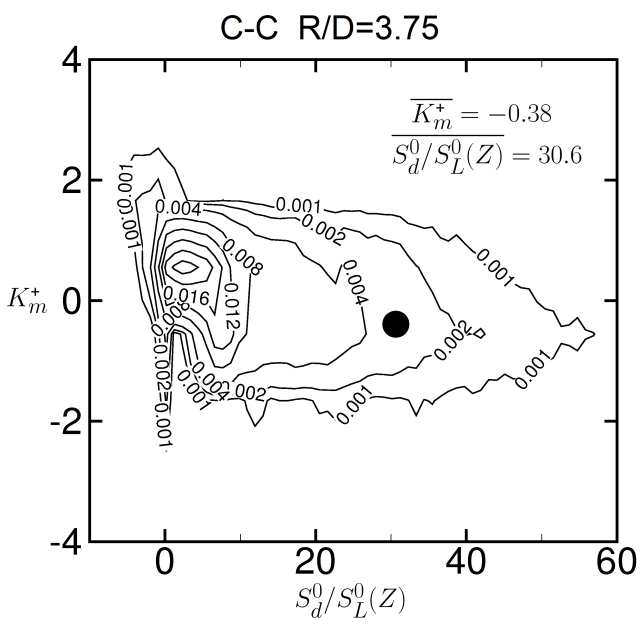

Figure 13: Joint PDFs of normalised curvature, $K_{m}^{+}$, and normalised total displacement speed, $S_{d}^{0} / S_{L}^{0}(\widetilde{Z})$, for $R / D=3.75$ at position C-C. Mean values noted are marked using a solid circle.

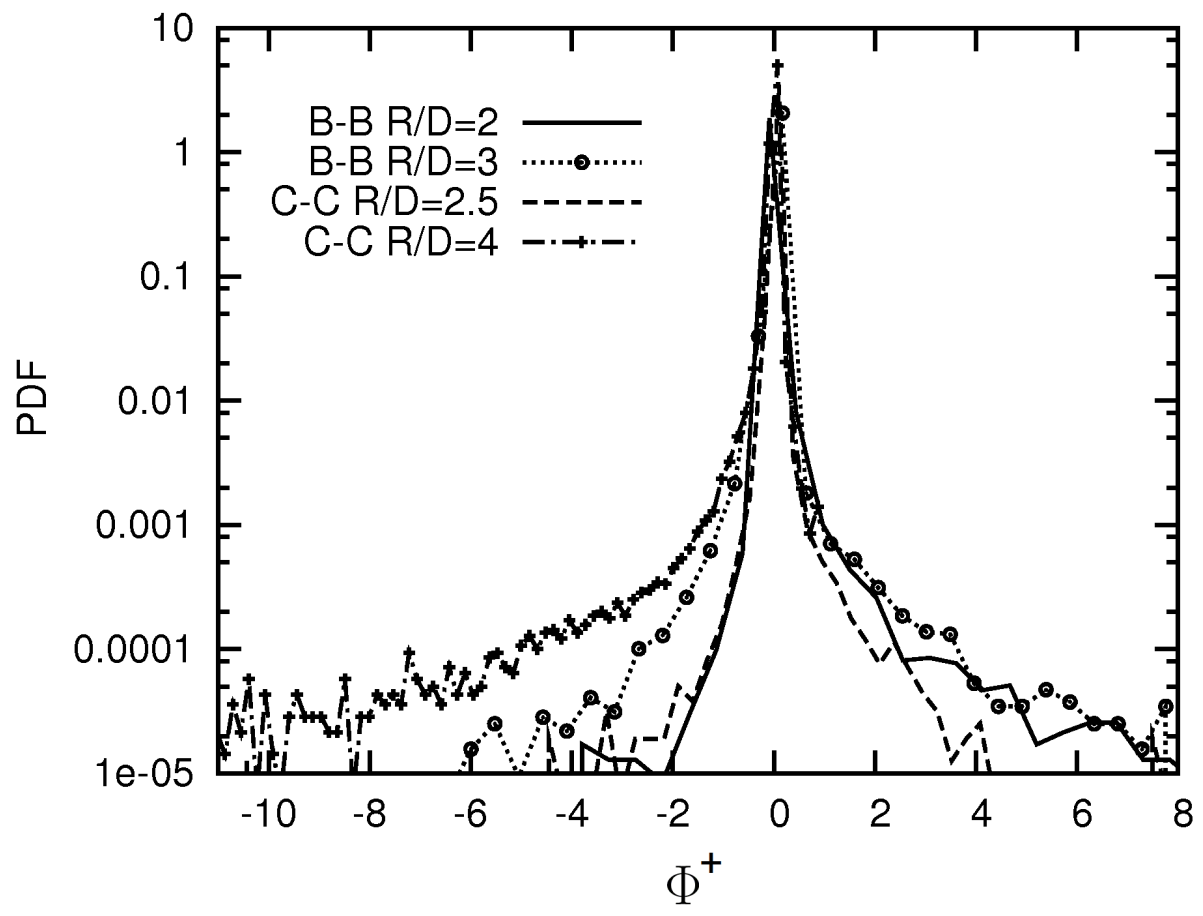

Figure 14: PDFs of normalised flame stretch, $\Phi^{+}=\Phi \delta_{L, s t}^{0} / S_{L, s t}^{0}$ for various axial and radial positions. 


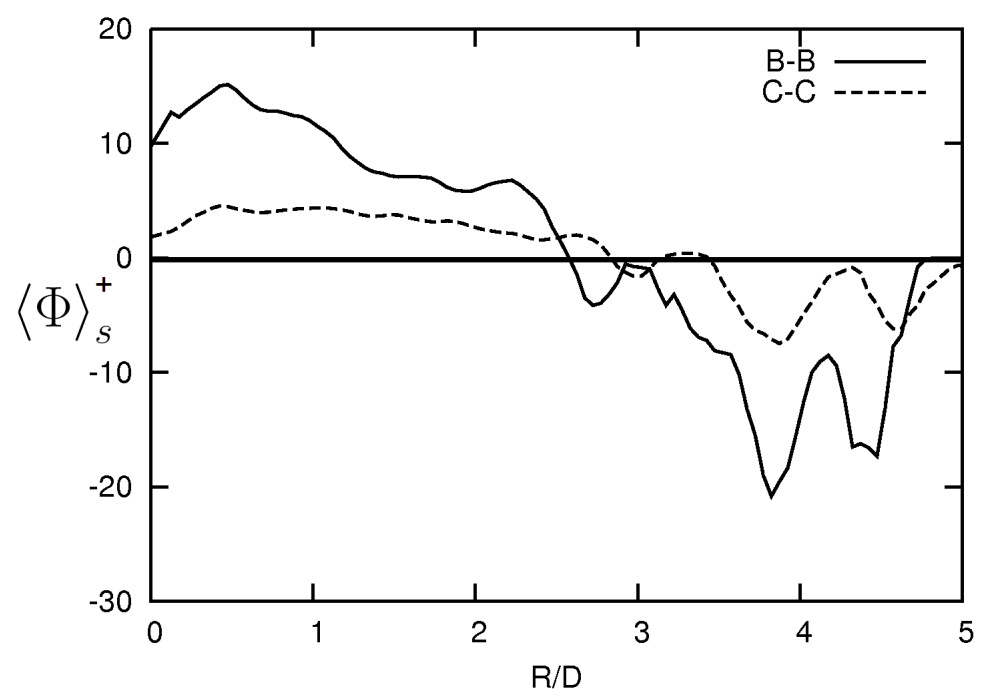

Figure 15: Radial variation of surface averaged flame stretch $\langle\Phi\rangle_{s}^{+}=\langle\Phi\rangle_{s} \delta_{L, s t}^{0} / S_{L, s t}^{0}$ for position $\mathrm{B}-\mathrm{B}$ and $\mathrm{C}-\mathrm{C}$.

(a)

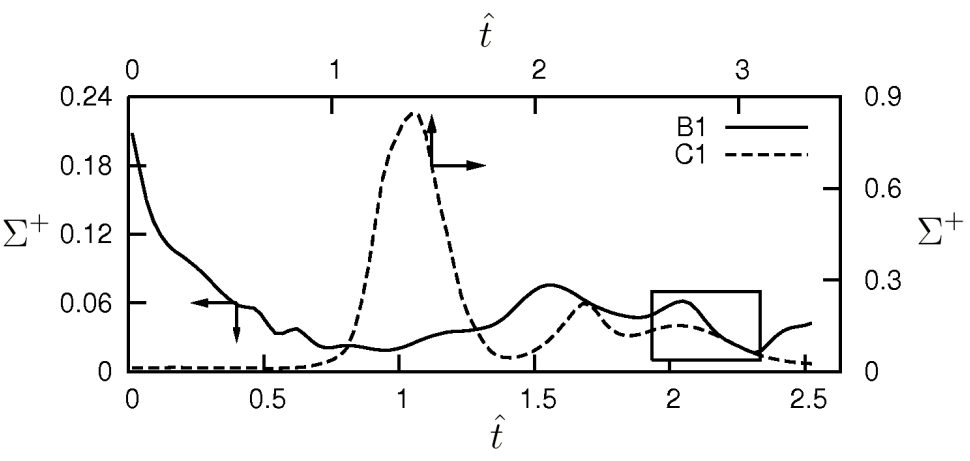

(b)

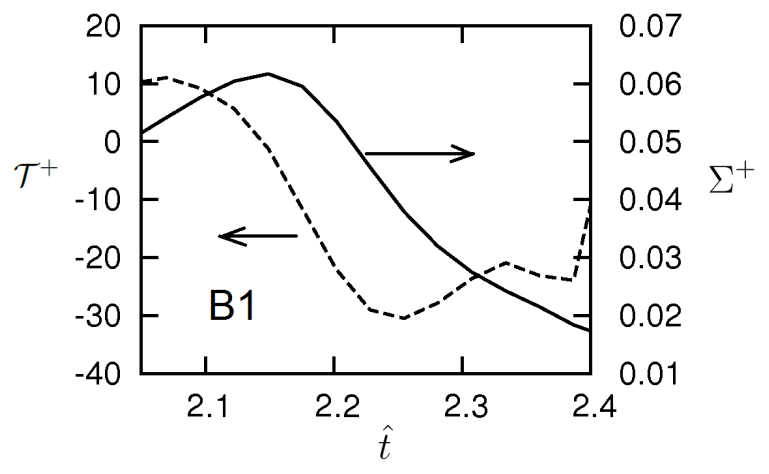

(c)

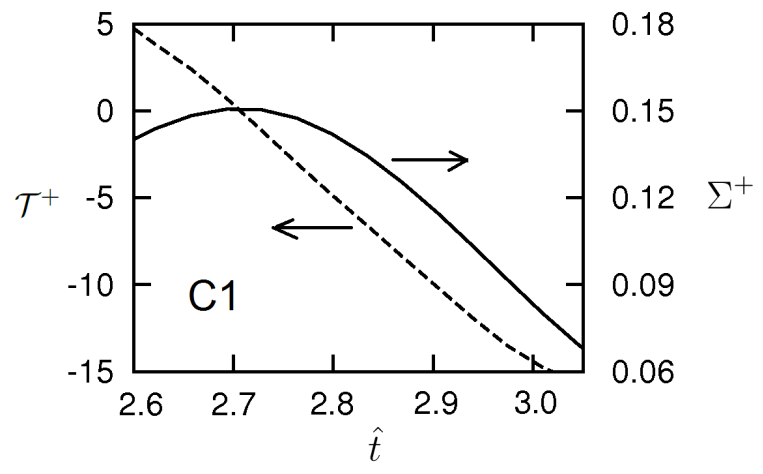

Figure 16: Variation of normalised FSD, $\Sigma^{+}=\Sigma \delta_{L}^{0}(\widetilde{Z})$ in (a), and its time derivative, $\mathcal{T}^{+}=\left(\partial \Sigma^{+} / \partial t\right) / \Sigma^{+}$at position $\mathbf{B 1}$ in (b) and $\mathbf{C 1}$ in (c). The variation of $\Sigma^{+}$over the entire simulation period is shown in (a) for the two locations. The time is normalised using the eddy turn over time at B1 and the time for the top $x$ axis of (a) is normalised using the eddy turn over time at $\mathbf{C 1}$. 

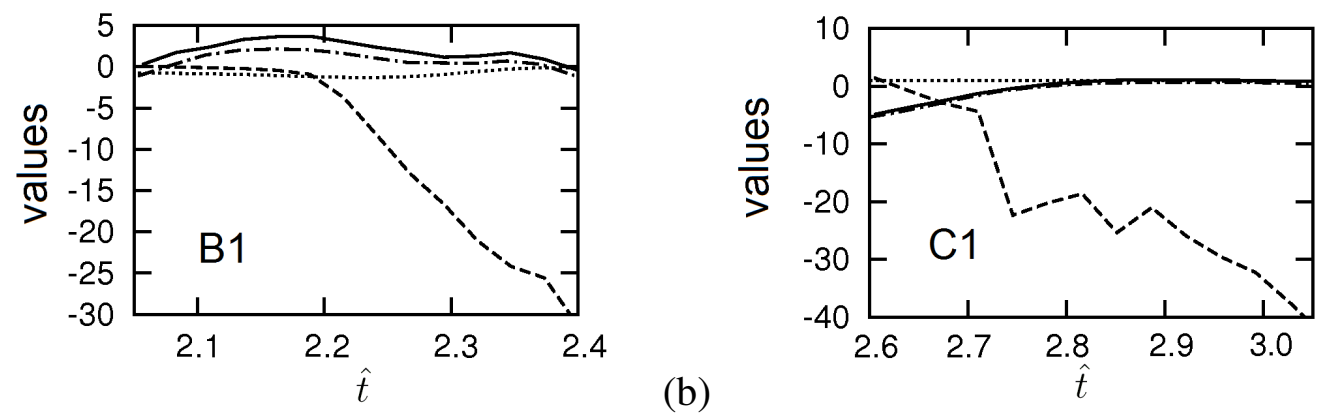

Figure 17: Temporal variation of normalised tangential strain rate, $\left\langle a_{T}\right\rangle_{s}^{+}$(solid line) curvature term $\left\langle 2 S_{d}^{*} K_{m}\right\rangle_{s}^{+}$(dashed), $\widehat{T_{1}^{+}}$(dash-dotted) and $\widehat{T_{2}^{+}}$(dotted) at positions (a) B1 and (b) C1. The normalisation is done using the local chemical time scale $t_{c}=$ $\delta_{L}^{0}(\widetilde{Z}) / S_{L}^{0}(\widetilde{Z})$. 